\title{
Contribution of TREM2 Signaling to the Development of Painful Diabetic Neuropathy by Mediating Microglial Polarization in Mice
}

xin chen

First People's Hospital of Foshan

Yue Le

First People's Hospital of Foshan

Si-qi Tang

First People's Hospital of Foshan

Wan-you He

First People's Hospital of Foshan

Jian He

First People's Hospital of Foshan

\section{Yun-hua Wang}

First People's Hospital of Foshan

Hanbing Wang ( $\nabla$ fswhbing@126.com )

First People's Hospital of Foshan https://orcid.org/0000-0003-0145-0081

\section{Research Article}

Keywords: TREM2, GLT-3, Microglia, Neuroinflammation, Painful diabetic neuropathy

Posted Date: October 26th, 2021

DOI: https://doi.org/10.21203/rs.3.rs-671471/v2

License: (9) This work is licensed under a Creative Commons Attribution 4.0 International License. Read Full License 


\section{Abstract}

Background: Painful diabetic neuropathy (PDN) is a common and intractable complication of diabetes mellitus, with little effective treatment. PDN has been associated with spinal neuroinflammation characterized by microglial activation. Recently, the triggering receptor expressed on myeloid cells 2 (TREM2), specifically localized on microglia, has been identified as a vital factor in modulating neuroinflammation and microglial phenotypes in neural diseases. Therefore, we hypothesized that spinal TREM2 might contribute to PDN and neuroinflammation by regulating microglial activity and phenotypes.

Methods $\llbracket$ Type I diabetes mellitus was elicited by a single intraperitoneal administration of streptozotocin (STZ) in mice. The pain behaviors were reflected by paw mechanical withdrawal thresholds (PMWT) and thermal withdrawal latency (PTWL).

Results $\llbracket$ We demonstrated that up-regulation of microglial TREM2 and amplification of both microglial $\mathrm{M} 1$ and $\mathrm{M} 2$ response was along with the presence of diabetes-related mechanical allodynia and thermal hypersensitivity. Moreover, we found that overexpression of TREM2 in microglia aggravated the symptom of PDN, amplified microglia M1 response, and suppressed microglia M2 polarization in the lumbar spinal cord of diabetic mice. However, inhibition of TREM2 with anti-TREM2 neutralizing antibodies attenuated mechanical allodynia and thermal hyperalgesia in diabetic mice. Besides, we identified Galectin-3 (GLT-3) as the potential ligand of the TREM2 receptor in facilitating the progression of PDN.

Conclusions: TREM2 could be a critical microglial membrane molecule that modulates microglial phenotypes pain hypersensitivity in PDN. GLT-3 might act as a specific ligand to trigger TREM2 signaling in PDN or other neuropathic pain.

\section{Introduction}

Painful diabetic neuropathy (PDN) is a common and intractable complication of diabetes, affecting approximately one in four diabetic patients[1]. The prevalence of PDN is even rising over time[2], owing to the growing diabetic population[3]. The typical clinical manifestation of PDN includes various unpleasant symptoms such as mechanical allodynia[4], substantially lowering life quality and increasing health care costs. Although opiates and antidepressants are applied to treat neuropathic pain, their effects on PDN are limited due to the intolerable side effects and poor response rates[$[5,6]$. Therefore, effective therapies specific to PDN are urgently needed.

Neuroinflammation in the spinal cord has been identified as an essential mechanism in neuropathic pain[7,8]. The hallmarks of neuroinflammation are the presence of activated microglia and reactive astrocytes that intensify the production of cytokines, chemokines, or neurotoxic compounds $[9,10]$. Studies on PDN also reveal a similar surge of neuroinflammation in the spinal cord and attribute these neuroinflammatory responses to microglia activation rather than astrocytes[11,12], suggesting a particular role of microglia in PDN. 
Although historically microglia were thought of as solely an inflammatory effector, multiple aspects of microglia are becoming established during the last decades. The activated microglia comprise many functional phenotypes, including the classically activated (M1) phenotype and the alternative activated (M2) phenotype[13,14]. In response to pathogens or tissue injury, the initially quiescent microglia transits into M1 microglia that release multiple pro-inflammatory cytokines or toxic substances in the early phase of inflammation[13,14]. In contrast, M2 microglia acquire an amoeboid morphology and obtain a promoted phagocytic function, allowing them to remove apoptotic neuronal debris and attenuate inflammation[13,14]. Studies have discovered the two opposing functional phenotypes of microglia in the spinal cord in the neuropathic pain induced by nerve injury[15] and bone cancer pain[16]. The neuropathic pain development was concomitant with the increase of M1 microglia, which express CD86 or iNOS, release pro-inflammatory cytokine such as IL-1 $\beta[17,18]$. However, M2 microglia expressing CD206 and arginase-1 (Arg-1) in the spinal cord was associated with increased anti-inflammatory mediators and neuropathic pain relief $[19,16]$. Thus, it is promising to relieve PDN by improving microglia polarization toward the M2 phenotype in the spinal cord.

The microglia functional plasticity depends on the transduction of stimulus signals through the cellsurface receptors. The triggering receptor expressed on myeloid cells 2 (TREM2), a cell-surface receptor predominantly expressed on microglia, has been implicated in numerous cerebral diseases, including Parkinson's disease (PD)[20,21], Alzheimer's disease (AD)[22,23], and ischemic brain injury[24,25]. Cells highly expressing TREM2 reportedly mediate immune surveillance and inflammation resolution[26]. By contrast, the deletion of TREM2 results in the impairment of phagocytic capacity and the release of proinflammatory cytokines[27]. Moreover, overexpression of TREM2 confers neuroprotection against Parkinson's disease[20], Alzheimer's disease[28], and cerebral ischemia/reperfusion injury[25] by promoting microglial M2 polarization. However, the conclusion on TREM2 should await because multiple studies provide evidence that inhibition of TREM2 or its downstream DNAX-activating protein of $12 \mathrm{kDa}$ (DAP12) could reduce the microglial number and pro-inflammatory cytokine production in the animal models of PD[29] (induced by 6-OHDA), traumatic brain injury[30], and peripheral nerve injury[31]. Therefore, the present study aimed to investigate the role of spinal TREM2 in the pathogenesis of PDN and its underlying mechanism related to microglia M1/M2 polarization.

In the present study, we reveal a detrimental role of TREM2 in exacerbating neuropathic pain and neuroinflammation in a mouse model of diabetic neuropathy. Our findings demonstrated that microglial TREM2 activation could aggravate neuroinflammation in the spinal cord by suppressing microglial M2 polarization. Furthermore, we also identified galectin-3 (GLT-3) as the specific ligand of TREM2 in facilitating the progression of PDN. The block of TREM2 or GLT-3 exerted potent analgesic and antiinflammatory effects in PDN.

\section{Methods}

\section{Animals}


Male C57BL/6J mice, weighing 22 25 g, were purchased from Laboratory Animal Center of Guangdong Province (Guangzhou, China), and kept in a standard lab housing with a 12-h light/dark cycle at a temperature of $21 \pm 2{ }^{\circ} \mathrm{C}$ and $60-70 \%$ humidity and allowed access to normal standard diet and water ad libitum.

\section{Induction of type 1 diabetes mellitus in mice}

Type 1 diabetes mellitus (T1DM) was induced by a single intraperitoneal (i.p.) injection of freshly prepared streptozotocin (STZ) (150 mg/kg; Sigma-Aldrich, Germany) in sterile $0.1 \mathrm{M}$ sodium citrate buffer (pH 4.5)[32]. Control mice were only injected i.p. with vehicle solution (sterile $0.1 \mathrm{M}$ sodium citrate buffer, $\mathrm{pH}$ 4.5). The successful establishment of T1DM was confirmed by determining the fasting blood glucose level with Accu-Chek test strips (Roche Diagnostics, Indianapolis, IN, U.S.A.) 3 days after the STZ injection. All the STZ-injected mice presented with high levels of blood glucose (>16.7 mmol/L). No mouse died through the study period or required insulin supplementation to offset extreme weight loss, and, at study end, all STZ-injected mice remained hyperglycemic (fasting blood glucose $>16.7 \mathrm{mmol} / \mathrm{L}$ ).

\section{Determination of mechanical withdrawal thresholds (PMWT) and thermal withdrawal latency (PTWL)}

On the experiment day, the mice were placed individually in transparent test compartments with a wire mesh bottom and habituated for one hour. Paw mechanical withdrawal thresholds (PMWTs) in response to mechanical stimuli were evaluated using the electronic von Frey unit (Bioseb, Montpellier, France) with a flexible metal filament applying increasing force (from 0 to $10 \mathrm{~g}$ ) against the plantar surface of the hind paw of the mouse[33]. The nocifensive paw withdrawal response automatically turned off the stimulus, and the mechanical pressure that evoked the response was recorded.

Thermal hyperalgesia was assessed by measuring paw thermal withdrawal latency (PTWL) to thermal stimuli using PL-200 Plantar Analgesia Tester (Chengdu Technology \& Market Co., Ltd., Sichuan, China) as described previously[34,35]. Briefly, the mice were placed on a glass plate and were allowed to adjust to the apparatus for $30 \mathrm{~min}$. The radiant heat lamp source was positioned right beneath the hind paw's plantar surface, vertically projecting a light spot with a diameter of $5 \mathrm{~mm}$. The PTWL was calculated by averaging three individual trials with 5-min intervals to prevent unexpected thermal sensitization. A cutoff time of $12 \mathrm{~s}$ was set to avoid tissue injury.

\section{Intrathecal administration of lentiviral vectors and neutralizing antibodies}

The intrathecal (i.t.) injection method was performed as previously described [36]. In brief, mice were covered with a soft towel and held gently but firmly by the hip bones via the thumb and index finger of the 
non-dominant hand of the operator. A $5 \mu \mathrm{L}$ microsyringe (Hamilton Company, Nevada, USA; 30-G needle) was inserted at the midline of the iliac crest between the lumbar 5 th and 6 th vertebrae. This region is considered to be the level of the cauda equina. A reflexive flick of the tail indicates the successful puncture of the dura. Because this reaction and muscle tone are essential reflections, i.t. injections were performed in conscious mice. After achieving a $90 \%$ to $95 \%$ success rate in training sessions, we started these experiments.

The lentiviral vector overexpressing mouse TREM2 gene (NCBI ID: NM_031254.3) [LV-Trem2, pLentaihCD11b-Trem2-P2A-EGFP-3xFlag-WPRE-pA(LTR)] under the microglial specific CD11b promoter and a control LV [LV-control, pLentai-hCD11b-P2A-EGFP-3xFlag-WPRE-pA(LTR)] were provided by Taitool Bioscience (Shanghai, China)[37]. The titer for LV-TREM2 was $4.35 \times 10^{9}$ transducing unit $(T U) / \mathrm{ml}$, while the LV-control was $7.16 \times 10^{9} \mathrm{TU} / \mathrm{ml}$. A week before STZ injection, LV-TREM2 or LV-control vectors $\left(5 \times 10^{7}\right.$ TU, $5 \mu l)$ were injected i.t. using a $5-\mu l$ Hamilton microsyringe.

The anti-TREM2 neutralizing antibody $(1 \mu \mathrm{g}, \# 1729-\mathrm{T} 2, \mathrm{R} \& \mathrm{D}$; a recombinant mouse TREM-2 Fc chimera protein, which specifically bind to TREM2 ligands) or isotype control antibody ( $1 \mu \mathrm{g}$, Mouse lgG Fc, \#31205, Thermo Fisher Scientific, Hampton, NH) was injected i.t. in a volume of $3 \mu$ l using a $5-\mu l$ Hamilton microsyringe (30-G needle) once a day for consecutive three days from D3 to D5 after STZ injection[38].

\section{Western blot}

The mice were sacrificed by anaesthetic overdose (2.5\% Avertin, $1600 \mathrm{mg} / \mathrm{kg}$, i.p.). The lumbar enlargement (L4-L5) of the spinal cord was rapidly removed and homogenized in ice-cold RIPA buffer (Beyotime, Shanghai, China). The lysates were kept for $30 \mathrm{~min}$ and then centrifuged at $14,000 \mathrm{~g}$ for 10 min at $4{ }^{\circ} \mathrm{C}$. Supernatants were collected, and the protein concentration was determined by the BCA protein assay kit (Boster, Wuhan, China). Equal amounts of protein samples (30 mg) from each group were separated using $10 \%$ sodium dodecyl sulphate-polyacrylamide gel electrophoresis and transferred onto polyvinylidene difluoride (Millipore, Bedford, MA, USA) membranes. Afterwards, the membranes were blocked with $5 \%$ BSA in TBST for $1 \mathrm{~h}$ at room temperature and then incubated with the primary antibodies overnight at $4{ }^{\circ} \mathrm{C}$. On the next day, the membranes were rinsed three times with TBST and incubated with secondary antibody (anti-mouse or rabbit lgG, 1:5000; Boster) for $2 \mathrm{~h}$ at room temperature. After washing three times with PBST, the protein bands were detected with enhanced chemiluminescent reagents (Boster) and analyzed by densitometric quantification using Fluochem HD2 Imaging System (Alpha Innotech, USA).

The following primary antibodies were used: anti-Apolipoprotein E (ApoE) (\#A0304, Abclonal, Wuhan, China; 1:500), anti-Arginase-1(Arg-1) (\#93668, Cell Signaling Technology, Beverly, MA; 1:500), anti-CD206 (\#ab64693, Abcam, Cambridge, MA; 1:1000), anti-CD86 (\#ab213044, Abcam; 1:1000), anti-DNAXactivating protein of $12 \mathrm{kDa}$ (DAP12) (\#12492, Cell Signaling Technology; 1:1000), anti-Galectin-3 (GLT-3) 
(\#A11198, Abclonal; 1:1000), anti-interlerkin (IL)-10 (\#A2171, Abclonal; 1:1000), anti-IL-1ß (\#A16288, Abclonal; 1:1000), anti-Transforming growth factor $\beta$ (TGF- $\beta$ ) (\#A18692, Abclonal; 1:1000), anti-TREM2 (\#PA5-87933, Thermo Fisher Scientific; 1:1000), and anti- $\beta$-actin (\#AC026, Abclonal; 1:10000). The experiments were carried out in triplicates ( $n=4$ or 5 /group).

\section{Immunofluorescence analysis}

For immunofluorescence analysis, mice were transcardially perfused with $4 \%$ iced formaldehyde ( $n=4$ or 5 /group). The lumbar spinal cord was then removed, embedded in paraffin, and cut into 5 - $\mu \mathrm{m}$ thick serial sections. After deparaffination, rehydration, heat-induced antigen retrieval with microwave oven (microwave method), the sections were incubated with 10\% (vol/vol) normal goat bovine serum for 60 min at room temperature and were then incubated overnight at $4{ }^{\circ} \mathrm{C}$ with primary antibodies against ApoE (\#A0304, rabbit polyclonal, Abclonal; 1:200), CD206 (\#ab64693, rabbit monoclonal, Abcam; 1:200), CD86 (\#ab213044, mouse monoclonal, Abcam; 1:200), GFAP (\#3670, mouse monoclonal, Cell Signaling Technology; 1:200), GLT-3 (\#A11198, rabbit monoclonal, Abclonal; 1:200), mouse lba-1 (\#MABN92, mouse monoclonal, Millipore, Billerica, MA; 1:200), rabbit lba-1 (\#17198, rabbit monoclonal, Cell Signaling Technology; 1:200), NeuN (\#ab104224, mouse monoclonal, Abcam; 1:200), and TREM2 (\#PA5-87933, rabbit polyclonal, Thermo Fisher Scientific; 1:200). The next day, the sections were washed by PBS and incubated for 60 min at room temperature with Dylight 488 (1:500; goat anti-rabbit; Abcam) or Dylight 594-labelled goat anti-rabbit secondary antibody (1:200; goat anti-rabbit; Abcam). Nuclei were counterstained with 4',6-diamidino-2-phenylindole (DAPI) (Boster). The images were acquired under immunofluorescence microscopy (Olympus, BX53; Melville, NY, USA), and the quantification of cell numbers was performed manually by counting the number of positive cells using the Image Pro ${ }^{\text {TM }}$ Plus software.

\section{Coimmunoprecipitation Assay}

Total protein extracts were prepared from the spinal cord as the Western blot, followed by homogenization with immunoprecipitation buffer (Beyotime) supplemented with $1 \mathrm{mM}$ PMSF protease inhibitor for 1 hour. After centrifugation at 12,000 rpm for 10 minutes, $200 \mathrm{mg}$ of the protein extract was incubated with $5 \mathrm{mg}$ of goat anti-TREM2 polyclonal antibody (Abcam) overnight at $4{ }^{\circ} \mathrm{C}$, followed by precipitation with protein A/G-agarose (Millipore, Billerica, Mass) for 3 hours[39]. After washing the immunoprecipitation buffer and heating at $95{ }^{\circ} \mathrm{C}$ for 10 minutes, the immunoprecipitates were subjected to SDS-PAGE and immunoblotting, followed by Densitometric quantification ( $n=4$ per group).

\section{Statistical analysis}

All data are presented as mean \pm standard deviations (SD). One-way ANOVA followed by Dunnett's multiple comparison test or two-way ANOVA followed by Bonferroni post hoc test was performed using 


\section{Results}

\section{The spinal TREM2 increases along with the development of PDN}

To induce the experimental type 1 diabetes (T1DM), we injected the mice i.p. with a single dose of STZ (150 mg/kg, Sigma). Then, we determined the behavioral changes in mice receiving STZ (T1DM) or saline injection (Saline). The results showed that compared with the Control group, the PMWTs of the STZ-injected mice began to decrease 3 days after STZ injection (D3) and reached the bottom on D14 (Fig. 1B, $F_{1,10}=144.4, P<0.0001, n=6$, two-way ANOVA; mean \pm SD: T1DM vs. Saline on D14, $2.70 \pm 0.33$ g vs. $5.12 \pm 0.33 \mathrm{~g}, n=6, P<0.0001$, Bonferroni post hoc analysis), indicating the presence of mechanical allodynia in the STZ-treated mice. We also tested the thermal nociception behaviors after STZ injection using a thermal paw stimulation system. The results suggested that, compared with the Control, the PTWL of the STZ-injected mice dropped after STZ injection, with the statistically significant difference on D7 and D14 (Fig. 1C, $F_{1,10}=43.93, P<0.0001, n=6$, two-way ANOVA; mean \pm SD: T1DM vs. Saline on D14, $1.95 \pm 0.22 \mathrm{~s} v s .3 .63 \pm 0.16 \mathrm{~s}, n=6, P<0.0001$, Bonferroni post hoc analysis), suggesting evident thermal hyperalgesia in the STZ-injected mice.

Next, we determined the pattern of TREM2 expression in the lumbar cord of the diabetic mice (T1DM) on baseline (BL), D7, and D14. The Western blot results showed that the protein expression of TREM2 and its downstream DAP-12 in the lumbar spinal cord markedly increased on D7 and D14 (Fig. 1D, $P=0.0492$ on $\mathrm{D} 7$ and $P=0.0007$ on $\mathrm{D} 14, n=5)$. These results indicated that the spinal TREM2 was activated along with the development of PDN.

The immunofluorescence double-staining results showed that on D14, TREM2 was mostly expressed in microglia in the spinal dorsal horn of STZ-treated mice (Fig. 1E). The statistical analysis indicated that the number of Iba-1+ microglia and TREM2+lba-1+ cells was minimal in the lumbar cord of the Control mice but substantially increased in the STZ-treated diabetic mice (Fig. 1F; mean \pm SD: T1DM vs. Saline on D14, lba-1+ microglia, $49.64 \pm 5.24 / \mathrm{mm}^{2} v s .10 .26 \pm 2.03 / \mathrm{mm}^{2}, P=0.0926, n=5$; Trem2+lba- $1+$ microglia, $\left.19.62 \pm 4.96 / \mathrm{mm}^{2} v s .1 .85 \pm 0.27 / \mathrm{mm}^{2}, P<0.0001, n=5\right)$.

\section{Both $\mathrm{M} 1$ and $\mathrm{M} 2$ microglia increase in parallel to the development of PDN}

The switch between M1 and M2 microglial phenotypes regulates the inflammatory response in the CNS[40]. Numerous studies have demonstrated that microglial activation, but not astrocytes, contributes to PDN[11,12]. Thus, we examined the protein expression of the M1 (CD86) and M2 (CD206 and Arg-1) microglia phenotype markers in the lumbar spinal cord. The results revealed that the protein levels of 
both M1 (CD86) and M2 (CD206 and Arg-1) microglia phenotype markers were markedly elevated on D7 and D14 after STZ injection (Fig. 2A-D, $P<0.05, P<0.01$, or $P<0.0001, n=5$ ). This observation was further verified by the immunofluorescence test, which indicated that the number of CD86+lba-1+and CD206+lba-1+ cells was significantly augmented in the lumbar spinal dorsal horn of the STZ-treated mice on D14 (Fig. 2E-G; mean \pm SD: T1DM vs. Saline on D14, CD86+lba- $1+$ microglia, $17.72 \pm 2.32 / \mathrm{mm}^{2} v s$. $2.73 \pm 1.90 / \mathrm{mm}^{2}, P<0.0001, n=5 ;$ CD $206+$ lba- $1+$ microglia, $7.56 \pm 2.19 / \mathrm{mm}^{2} v s .1 .20 \pm 1.13 / \mathrm{mm}^{2}$, $P=0.0004, n=5)$. We also determined the expression of IL-1 $\beta$, TGF- $\beta$, and IL-10 in the spinal cord, as IL-1 $\beta$ is derived explicitly from M1 phenotype microglia, while the primary source of TGF- $\beta$ and IL-10 is M2 phenotype. We found that the spinal protein levels of IL-1 $\beta$, TGF- $\beta$, and IL-10 were increased on D7 and D14 after STZ injection (Fig. 2H-K, $P<0.05, P<0.01$, or $P<0.0001, n=5$ ).

\section{Overexpression of TREM2 in microglia aggravates PDN in mice}

Previous studies have demonstrated that TREM2 inhibits neuroinflammatory response in neurodegenerative diseases such as Alzheimer's disease[22,23], and this effect may be related to its promotion of M2 phenotype microglia. Given the anti-inflammatory properties of M2 phenotype microglia and the above results, we speculated that overexpression of the TREM2 gene in microglia might alleviate PDN by suppressing spinal inflammation. In this regard, we injected intrathecally (i.t.) the mice with

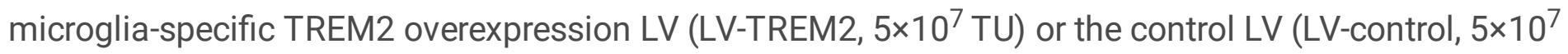
TU, 7 days before STZ injection) 7 days before STZ injection.

We found that the injection of LV-control did not alter the PMWTs and PTWLs compared with T1DM mice that only received an i.t. injection of saline (Fig.3 A and B; T1DM vs. T1DM+LV-control: PMWT, $F_{1,14}=$ $2.543, P>0.05, n=8$, two-way ANOVA; PTWL, $F_{1,14}=2.372, P>0.05, n=8$, two-way ANOVA). In contrast to our expectation, the injection of LV-TREM2 decreased the PMWTs and extended the PTWLs of the T1DM+LV-TREM2 mice (Fig. $3 \mathrm{~A}$ and B; T1DM vs. T1DM+LV-TREM2: PMWT, $F_{1,14}=35.21, P<0.0001, n=8$, two-way ANOVA; PTWL, $F_{1,14}=14.27, P=0.0020, n=8$, two-way ANOVA), while the protein expression of TREM2 in the lumbar spinal cord was elevated (Fig. 3D, $P<0.0001, n=4$ ).

This unexpected result was confirmed by the immunofluorescence test, which showed that the injection of LV-TREM2 amplified the number of Iba-1 microglia and CD86+lba-1+ cells (M1 phenotype), but decreased the CD206+lba-1+ cells (M2 phenotype) in the spinal dorsal horn (Fig.3 E-H; mean \pm SD, T1DM vs. T1DM+LV-TREM2 on D14: Iba-1+ microglia, $36.83 \pm 7.25 / \mathrm{mm}^{2} v s .65 .17 \pm 10.29 / \mathrm{mm}^{2}, P=0.0002, n=4$; CD86+lba-1+ microglia, $12.98 \pm 1.25 / \mathrm{mm}^{2}$ vs. $17.20 \pm 2.89 / \mathrm{mm}^{2}, P=0.0261, n=4 ;$ CD206+lba- $1+$ microglia, $9.44 \pm 3.01 / \mathrm{mm}^{2} v s$. $\left.2.79 \pm 1.14 / \mathrm{mm}^{2}, P=0.0032, n=4\right)$. Furthermore, the protein expression of pro-inflammatory cytokine IL-1 $\beta$ was increased by the injection of LV-TREM2, whereas anti-inflammatory cytokines TGF- $\beta$ and IL-10 were reduced (Fig.3I, $P<0.05$ or $P<0.001$ compared with T1DM, $n=5$ ). Together, 


\section{Intrathecal treatment with anti-TREM2 neutralizing antibody ameliorates PDN in mice}

Based on the above data, it was reasonable to argue that inhibition of TREM2 signalling might relieve PDN. Next, we investigate whether inhibition of the spinal TREM2 with a TREM2 neutralizing antibody can attenuate the pain-like behavior in T1DM mice. The behavioral test showed that the i.t. treatment with TREM2 neutralizing antibody ( $1 \mu \mathrm{g} / \mathrm{d}$, from D3 to D5 after STZ injection, i.t.) elevated the PMWTs and TWLs in the T1DM+anti-TREM2 mice as compared with the T1DM mice receiving i.t. injection of saline (Fig. $4 \mathrm{~B}$ and C; T1DM vs. T1DM+anti-TREM2: PMWT, $F_{1,16}=23.17, P=0.0002, n=9$, two-way ANOVA; PTWL, $F_{1,16}=19.65, P=0.0004, n=9$, two-way ANOVA), while the IgG-treated T1DM mice did not present with any behavioral change (Fig. $4 \mathrm{~B}$ and C; T1DM vs. T1DM+lgG: PMWT, $F_{1,16}=0.8287, P=0.3762, n=9$, two-way ANOVA; PTWL, $F_{1,16}=0.1968, P=0.6632, n=9$, two-way ANOVA).

The immunofluorescence results showed that the injection of TREM2 neutralizing antibody reduced the spinal number of Iba-1 microglia and CD86+lba-1+ cells (M1 phenotype) and increased the CD206+lba-1+ cells (M2 phenotype) in the spinal dorsal horn of T1DM+anti-TREM2 mice (Fig.4 D-G; mean \pm SD, T1DM vs. T1DM+anti-TREM2 on D14: CD86+lba- $1+$ microglia, $14.51 \pm 2.01 / \mathrm{mm}^{2} v s .8 .21 \pm 2.87 / \mathrm{mm}^{2}$, $P=0.0024, n=4$; CD206+lba-1+ microglia, $7.34 \pm 0.80 / \mathrm{mm}^{2} v s .10 .08 \pm 1.48 / \mathrm{mm}^{2}, P=0.0052, n=4$ ). Furthermore, the protein level of pro-inflammatory cytokine IL-1 $\beta$ was decreased by the injection of TREM2 neutralizing antibody, whereas anti-inflammatory cytokines TGF- $\beta$ and IL-10 were increased (Fig. $4 \mathrm{H}-\mathrm{K}, P<0.01$ or $P<0.001$ compared with T1DM, $n=5)$.

\section{GLT-3 is the potential ligand of the microglial TREM2 receptor in facilitating the progression of PDN}

Furthermore, we asked what ligand triggered the TREM2 signalling in the development of PDN. Recent studies suggested that ApoE[41,42] and GLT-3[43] were the potential ligands of TREM2 in mediating a series of downstream actions. Therefore, we detected the protein expression of ApoE and GLT-3 in the lumbar spinal cord of the T1DM mice. We found that the spinal protein expression of GLT-3, but not ApoE, was significantly increased in the STZ-treated diabetic mice on D7 and D14 after STZ injection (Fig. 5 AC; ApoE, $P=0.9248$ on D7, $P=0.6238$ on D14, $n=5$; GLT-3, $P=0.0288$ on D7, $P<0.0001$ on $\mathrm{D} 14, n=5)$.

To corroborate these proteins' attachment with the TREM2 receptor, we performed immunoprecipitation by antibodies against TREM2 and tested its efficiency using western blot analysis. We found that the immunoprecipitated GLT-3 with TREM2 antibodies from the lumbar spinal cord of diabetic mice on D14 
after STZ injection was more than that of saline-treated mice (Fig. 5D), while the difference in the blotted ApoE was not found (Fig. 5D).

We also identified the colocalization of ApoE or GLT-3 with the immunofluorescence double-staining procedure. We observed that in the spinal dorsal horn, ApoE expression was primarily colocalized with neurons (NeuN+) and astrocyte (GFAP+) (Fig. 5E), while GLT-3 expression was largely overlapped with Iba-1+ microglia (Fig. 5F).

Next, we examine whether neutralizing GLT-3 in the spinal cord can affect the behavioral manifestation of PDN and the microglial M1/M2 balance. The behavioral data showed that the injection of GLT-3 neutralizing antibody ( $1 \mu \mathrm{g} / \mathrm{d}$, from D3 to D5 after STZ injection, i.t.) increased the PMWTs and PTWLs in the T1DM+anti-GLT-3 mice (Fig. $6 \mathrm{~B}$ and C; T1DM vs. T1DM+anti-GLT-3: PMWT, $F_{1,16}=16.09, P=0.001$, $n=9$, two-way ANOVA; PTWL, $F_{1,16}=40.63, P<0.0001, n=9$, two-way ANOVA). The immunofluorescence assays suggested that the i.t. injection of GLT-3 neutralizing antibody reduced the spinal number of Iba-1 microglia and CD86+lba-1+ cells (M1 phenotype) and increased the CD206+lba-1+ cells (M2 phenotype) in the spinal dorsal horn of T1DM+anti-GLT-3 mice (Fig. 6 D-G; mean \pm SD, T1DM vs. T1DM+anti-TREM2 on D14: CD86+lba-1+ microglia, $15.34 \pm 1.40 / \mathrm{mm}^{2}$ vs. $6.60 \pm 2.42 / \mathrm{mm}^{2}, P<0.0001, n=4 ;$ CD206+lba-1+ microglia, $\left.7.34 \pm 1.47 / \mathrm{mm}^{2} v s .11 .66 \pm 3.46 / \mathrm{mm}^{2}, P=0.0301, n=4\right)$. Furthermore, the protein level of proinflammatory cytokine IL-1 $\beta$ was decreased by the injection of GLT-3 neutralizing antibody, whereas antiinflammatory cytokines TGF- $\beta$ and IL-10 were increased (Fig. $6 \mathrm{H}-\mathrm{K}, P<0.05, P<0.01$, or $P<0.001, n=5$ ).

\section{Discussion}

In the present study, we investigated the role of TREM2 in the development of PDN and explored its underlying mechanism in mice. We demonstrated that up-regulation of microglial TREM2 and increase of both microglial M1 and M2 response was along with the presence of diabetes-related mechanical allodynia and thermal hyperalgesia. Moreover, we found that overexpression of TREM2 in microglia aggravated the symptom of PDN, amplified microglia M1 response, and suppressed microglia M2 polarization in the lumbar spinal cord of diabetic mice. However, inhibition of TREM2 with anti-TREM2 neutralizing antibodies attenuated mechanical allodynia and thermal hyperalgesia in diabetic mice. Besides, we identified GLT-3 as the potential ligand of the TREM2 receptor in facilitating the progression of PDN.

Neuroinflammation, an immune response that arises in the central nervous system (CNS), has been recognized as a hallmark of many neurologic conditions[44,45], including PDN[46,47] and other diabetic cerebral disorders[48]. A critical role of neuroinflammation is also found in neuropathic pain, characterized by specific activation of microglia rather than astrocytes and increased release of various cytokines[49]. Studies on PDN also reveal a similar surge of neuroinflammation in the spinal cord and attribute these neuroinflammatory responses to microglia activation rather than astrocytes[11,12], suggesting a particular role of microglia in PDN. As a result, investigating the mechanism underlying 
microglial activation, especially the specific receptors expressed on the microglial surface, is of great potential for treating neuropathic pain.

In the CNS, TREM2 is a transmembrane receptor predominantly expressed on microglia[20,21]. However, the role of TREM2 in modulating neuroinflammation is still controversial. A large body of studies in neural diseases (i.e., $A D[22,23]$ and $P D[20,21]$ ) suggested a beneficial effect of TREM2 by decreasing neuroinflammation and aggravating cognitive impairment. On the other hand, some studies reported an opposite deleterious effect of microglial TREM2 by facilitating neuroinflammation. Another research also recently associated TREM2 with diabetic brain disorder by showing that overexpression of TREM2 alleviated neuroinflammatory response and cognitive decline in Type 2 diabetes mellitus mice[50]. These results led us to clarify the role of TREM2 in PDN.

Our results showed that TREM2 protein levels and microglial numbers simultaneously increase after the induction of T1DM with STZ injection. Since the fold changes of the protein expression in the dorsal horn were comparable to those of microglial numbers, the increase in TREM2 may be due to an increased number of microglia rather than up-regulated expression. Our results indicated that overexpression of microglial TREM2 augmented the spinal inflammatory response (production of pro-inflammatory cytokines IL-1 $\beta$ and TNF- $a$ ) and exacerbate pain hypersensitivity in PDN mice. Conversely, inhibiting TREM2 with anti-TREM2 neutralizing antibody decreased the spinal neuroinflammation and ameliorated the pain behavior. This finding is consistent with previous studies on peripheral nerve injury-induced neuropathic pain[31,51], demonstrating that DAP12 (downstream of TREM2) knockout suppresses spinal pro-inflammatory responses.

Although previous studies have unravelled the critical role of microglia in PDN, most consider microglia as a marker of inflammation (M1 phenotype) and ignore the M2 phenotype. TREM2 is thought to be an essential molecule that regulates microglial phenotype and activation status. We found that M1 and M2 microglia increased in the spinal cord of PDN mice, and the increase of spinal microglial number was largely M1 microglia[50]. This finding is partly consistent with a recent study[50], which also observed a rise in M1 and M2 microglia in T2DM mice. However, they found overexpression of TREM2 promoted microglial M2 polarization, whereas we suggested that overexpression of microglial TREM2 increased M1 response but reduced microglial M2 phenotypes. The phenomena were further supported by the result that treatment with anti-TREM2 neutralizing antibody decreased M1 polarization and enhanced M2 polarization.

Together, TREM2-mediated signals could be one of the critical pathways in microglial M1/M2 polarization.

Although the ligand that binds to and activates TREM2 remains elusive, a broad array of lipids produced directly or indirectly due to damage to primary sensory neurons has been proposed as potential TREM2 ligands. TREM2 could serve as a microglial sensor for lipid mediators released from damaged sensory nerve terminals or other cells in the $\mathrm{DH}$. Recently, apolipoprotein $\mathrm{E}$ (ApoE) was reported as a potent ligand for TREM2. These reports suggest that ApoE could be a potent activator of the TREM2/DAP12 system in 
the present pain model. Furthermore, a recent study reveals Galectin-3 as a novel endogenous TREM2 ligand, which adversely regulates inflammatory response in $A D[43]$. Our results demonstrated that the protein expression of galectin-3 but not ApoE increased in the spinal cord of PDN mice. Furthermore, we observed that the immunoprecipitated GLT-3 with TREM2 antibodies was also elevated, indicating the GLT-3 might be the ligand for TREM2 signaling in PDN.

In conclusion, this study provides evidence that TREM2 could be a critical microglial membrane molecule that modulates microglial phenotypes and elicits pain hypersensitivity in PDN. Our results demonstrated that GLT-3 might act as a specific ligand to trigger TREM2 signaling in PDN or other neuropathic pain. GLT-3 and TREM2 could serve as potential therapeutic targets for PDN.

\section{Abbreviations}

APOE, apolipoprotein E; Arg-1, arginase-1; BL, baseline; CNS, central nerve system; DAPI, 4',6-diamidino-2phenylindole; GFAP, glial fibrillary acidic protein; GLT-3, galectin-3; Iba-1, ionized calcium-binding adaptor protein-1; IL-1 $\beta$, interleukin 1 $\beta$; IL-10, interleukin 10; LV-control, control lentiviral vectors; LV-TREM2, microglia specific TREM2 overexpression lentiviral vectors; ns, not significant; PDN, painful diabetic neuropathy; PMWT, paw mechanical withdrawal threshold; PTWL, paw thermal withdrawal latency; STZ, streptozotocin; T1DM, type 1 diabetes mellitus; TGF- $\beta$, transforming growth factor $\beta$; TREM2, triggering receptor expressed on myeloid cells 2 .

\section{Declarations}

\section{Acknowledgments}

This work was supported by grants from the National Natural Science Foundation of China (Grant No. 81771357), the Natural Science Foundation of Guangdong Province (Grant No. 2017A030313587), and the GuangDong Basic and Applied Basic Research Foundation (Grant No. 2020A1515110863).

\section{Funding}

This work was supported by National Natural Science Foundation of China (Grant No. 81771357), Natural Science Foundation of Guangdong Province (Grant No. 2017A030313587), and GuangDong Basic and Applied Basic Research Foundation (Grant No. 2020A1515110863).

\section{Competing interests}

The authors declare that they have no competing interests.

\section{Availability of data and materials}

The datasets during and/or analyzed during the current study are available from the corresponding author on reasonable request. 


\section{Authors' contributions}

X. C. and H.B.W. conceived the study, designed the experiments and wrote the manuscript. X. C., S.Q.T. and Y.L. performed most of the experiments. W.Y.H. and J.H. performed the intrathecal injection of drugs. Y.H.W, X. C., S.Q.T. and Y.L. performed all blind pain behavior tests.

\section{Ethics approval and consent to participate}

All procedures were strictly performed in accordance with the regulations of the ethics committee of the International Association for the Study of Pain and the Guide by Guangdong Medical Laboratory Animal Center. All animal experiments were approved by the National Institutes of Health and the institutional animal ethical committee and were designed to minimize suffering and the number of animals used.

\section{Consent for publication}

Not applicable.

\section{Acknowledgements}

Not applicable.

\section{References}

1. Diagnosis and classification of diabetes mellitus (2013). Diabetes Care 36 Suppl 1 (Suppl 1):S67-74. doi:10.2337/dc13-S067

2. Feldman EL, Callaghan BC, Pop-Busui R, Zochodne DW, Wright DE, Bennett DL, Bril V, Russell JW, Viswanathan V (2019) Diabetic neuropathy. Nature reviews Disease primers 5 (1):41. doi:10.1038/s41572-019-0092-1

3. Zimmet PZ, Magliano DJ, Herman WH, Shaw JE (2014) Diabetes: a 21 st century challenge. Lancet Diabetes Endocrinol 2 (1):56-64. doi:10.1016/s2213-8587(13)70112-8

4. Schreiber AK, Nones CF, Reis RC, Chichorro JG, Cunha JM (2015) Diabetic neuropathic pain: Physiopathology and treatment. World J Diabetes 6 (3):432-444. doi:10.4239/wjd.v6.i3.432

5. Bril V, England J, Franklin GM, Backonja M, Cohen J, Del Toro D, Feldman E, Iverson DJ, Perkins B, Russell JW, Zochodne D (2011) Evidence-based guideline: Treatment of painful diabetic neuropathy: report of the American Academy of Neurology, the American Association of Neuromuscular and Electrodiagnostic Medicine, and the American Academy of Physical Medicine and Rehabilitation. Neurology 76 (20):1758-1765. doi:10.1212/WNL.0b013e3182166ebe

6. Finnerup NB, Attal N, Haroutounian S, McNicol E, Baron R, Dworkin RH, Gilron I, Haanpää M, Hansson P, Jensen TS, Kamerman PR, Lund K, Moore A, Raja SN, Rice AS, Rowbotham M, Sena E, Siddall P, Smith BH, Wallace M (2015) Pharmacotherapy for neuropathic pain in adults: a systematic review and meta-analysis. Lancet Neurol 14 (2):162-173. doi:10.1016/s1474-4422(14)70251-0 
7. Teixeira-Santos L, Albino-Teixeira A, Pinho D (2020) Neuroinflammation, oxidative stress and their interplay in neuropathic pain: Focus on specialized pro-resolving mediators and NADPH oxidase inhibitors as potential therapeutic strategies. Pharmacol Res 162:105280. doi:10.1016/j.phrs.2020.105280

8. Bannister K, Sachau J, Baron R, Dickenson AH (2020) Neuropathic Pain: Mechanism-Based Therapeutics. Annu Rev Pharmacol Toxicol 60:257-274. doi:10.1146/annurev-pharmtox-010818021524

9. Schetters STT, Gomez-Nicola D, Garcia-Vallejo JJ, Van Kooyk Y (2017) Neuroinflammation: Microglia and T Cells Get Ready to Tango. Front Immunol 8:1905. doi:10.3389/fimmu.2017.01905

10. Liddelow SA, Guttenplan KA, Clarke LE, Bennett FC, Bohlen CJ, Schirmer L, Bennett ML, Münch AE, Chung WS, Peterson TC, Wilton DK, Frouin A, Napier BA, Panicker N, Kumar M, Buckwalter MS, Rowitch DH, Dawson VL, Dawson TM, Stevens B, Barres BA (2017) Neurotoxic reactive astrocytes are induced by activated microglia. Nature 541 (7638):481-487. doi:10.1038/nature21029

11. Wang D, Couture R, Hong Y (2014) Activated microglia in the spinal cord underlies diabetic neuropathic pain. Eur J Pharmacol 728:59-66. doi:10.1016/j.ejphar.2014.01.057

12. Tsuda M (2016) Microglia in the spinal cord and neuropathic pain. J Diabetes Investig 7 (1):17-26. doi:10.1111/jdi.12379

13. Orihuela R, McPherson CA, Harry GJ (2016) Microglial M1/M2 polarization and metabolic states. $\mathrm{Br}$ J Pharmacol 173 (4):649-665. doi:10.1111/bph.13139

14. Tang Y, Le W (2016) Differential Roles of M1 and M2 Microglia in Neurodegenerative Diseases. Mol Neurobiol 53 (2):1181-1194. doi:10.1007/s12035-014-9070-5

15. Piotrowska A, Kwiatkowski K, Rojewska E, Makuch W, Mika J (2016) Maraviroc reduces neuropathic pain through polarization of microglia and astroglia - Evidence from in vivo and in vitro studies. Neuropharmacology 108:207-219. doi:10.1016/j.neuropharm.2016.04.024

16. Huo W, Zhang Y, Liu Y, Lei Y, Sun R, Zhang W, Huang Y, Mao Y, Wang C, Ma Z, Gu X (2018) Dehydrocorydaline attenuates bone cancer pain by shifting microglial M1/M2 polarization toward the M2 phenotype. Mol Pain 14:1744806918781733. doi:10.1177/1744806918781733

17. Li Z, Wei H, Piirainen S, Chen Z, Kalso E, Pertovaara A, Tian L (2016) Spinal versus brain microglial and macrophage activation traits determine the differential neuroinflammatory responses and analgesic effect of minocycline in chronic neuropathic pain. Brain Behav Immun 58:107-117. doi:10.1016/j.bbi.2016.05.021

18. Kobiela Ketz A, Byrnes KR, Grunberg NE, Kasper CE, Osborne L, Pryor B, Tosini NL, Wu X, Anders JJ (2017) Characterization of Macrophage/Microglial Activation and Effect of Photobiomodulation in the Spared Nerve Injury Model of Neuropathic Pain. Pain Med 18 (5):932-946. doi:10.1093/pm/pnw144

19. Gong X, Chen Y, Fu B, Jiang J, Zhang M (2017) Infant nerve injury induces delayed microglial polarization to the $\mathrm{M} 1$ phenotype, and exercise reduces delayed neuropathic pain by modulating microglial activity. Neuroscience 349:76-86. doi:10.1016/j.neuroscience.2017.02.051 
20. Zhang Y, Feng S, Nie K, Li Y, Gao Y, Gan R, Wang L, Li B, Sun X, Wang L, Zhang Y (2018) TREM2 modulates microglia phenotypes in the neuroinflammation of Parkinson's disease. Biochem Biophys Res Commun 499 (4):797-802. doi:10.1016/j.bbrc.2018.03.226

21. Ren M, Guo Y, Wei X, Yan S, Qin Y, Zhang X, Jiang F, Lou H (2018) TREM2 overexpression attenuates neuroinflammation and protects dopaminergic neurons in experimental models of Parkinson's disease. Exp Neurol 302:205-213. doi:10.1016/j.expneurol.2018.01.016

22. Hickman SE, El Khoury J (2014) TREM2 and the neuroimmunology of Alzheimer's disease. Biochem Pharmacol 88 (4):495-498. doi:10.1016/j.bcp.2013.11.021

23. Ulrich JD, Ulland TK, Colonna M, Holtzman DM (2017) Elucidating the Role of TREM2 in Alzheimer's Disease. Neuron 94 (2):237-248. doi:10.1016/j.neuron.2017.02.042

24. Wu R, Li X, Xu P, Huang L, Cheng J, Huang X, Jiang J, Wu LJ, Tang Y (2017) TREM2 protects against cerebral ischemia/reperfusion injury. Mol Brain 10 (1):20. doi:10.1186/s13041-017-0296-9

25. Zhai Q, Li F, Chen X, Jia J, Sun S, Zhou D, Ma L, Jiang T, Bai F, Xiong L, Wang Q (2017) Triggering Receptor Expressed on Myeloid Cells 2, a Novel Regulator of Immunocyte Phenotypes, Confers Neuroprotection by Relieving Neuroinflammation. Anesthesiology 127 (1):98-110. doi:10.1097/aln.0000000000001628

26. Takahashi K, Rochford CD, Neumann H (2005) Clearance of apoptotic neurons without inflammation by microglial triggering receptor expressed on myeloid cells-2. J Exp Med 201 (4):647-657. doi:10.1084/jem.20041611

27. Piccio L, Buonsanti C, Mariani M, Cella M, Gilfillan S, Cross AH, Colonna M, Panina-Bordignon P (2007) Blockade of TREM-2 exacerbates experimental autoimmune encephalomyelitis. Eur J Immunol 37 (5):1290-1301. doi:10.1002/eji.200636837

28. Wang Y, Lin Y, Wang L, Zhan H, Luo X, Zeng Y, Wu W, Zhang X, Wang F (2020) TREM2 ameliorates neuroinflammatory response and cognitive impairment via PI3K/AKT/FoxO3a signaling pathway in Alzheimer's disease mice. Aging (Albany N Y) 12 (20):20862-20879. doi:10.18632/aging.104104

29. Virgone-Carlotta A, Uhlrich J, Akram MN, Ressnikoff D, Chretien F, Domenget C, Gherardi R, Despars G, Jurdic P, Honnorat J, Nataf S, Touret M (2013) Mapping and kinetics of microglia/neuron cell-to-cell contacts in the 6-OHDA murine model of Parkinson's disease. Glia 61 (10):1645-1658. doi:10.1002/glia.22546

30. Saber M, Kokiko-Cochran O, Puntambekar SS, Lathia JD, Lamb BT (2017) Triggering Receptor Expressed on Myeloid Cells 2 Deficiency Alters Acute Macrophage Distribution and Improves Recovery after Traumatic Brain Injury. J Neurotrauma 34 (2):423-435. doi:10.1089/neu.2016.4401

31. Kobayashi M, Konishi H, Sayo A, Takai T, Kiyama H (2016) TREM2/DAP12 Signal Elicits Proinflammatory Response in Microglia and Exacerbates Neuropathic Pain. J Neurosci 36 (43):11138-11150. doi:10.1523/JNEUROSCI.1238-16.2016

32. Furman BL (2015) Streptozotocin-Induced Diabetic Models in Mice and Rats. Curr Protoc Pharmacol 70:5.47.41-45.47.20. doi:10.1002/0471141755.ph0547s70 
33. Sałat K, Kołaczkowski M, Furgała A, Rojek A, Śniecikowska J, Varney MA, Newman-Tancredi A (2017) Antinociceptive, antiallodynic and antihyperalgesic effects of the 5-HT(1A) receptor selective agonist, NLX-112 in mouse models of pain. Neuropharmacology 125:181-188.

doi:10.1016/j.neuropharm.2017.07.022

34. Zhang MT, Wang B, Jia YN, Liu N, Ma PS, Gong SS, Niu Y, Sun T, Li YX, Yu JQ (2017) Neuroprotective effect of liquiritin against neuropathic pain induced by chronic constriction injury of the sciatic nerve in mice. Biomed Pharmacother 95:186-198. doi:10.1016/j.biopha.2017.07.167

35. Hargreaves K, Dubner R, Brown F, Flores C, Joris J (1988) A new and sensitive method for measuring thermal nociception in cutaneous hyperalgesia. Pain 32 (1):77-88. doi:10.1016/03043959(88) 90026-7

36. Hylden JL, Wilcox GL (1980) Intrathecal morphine in mice: a new technique. European journal of pharmacology 67 (2-3):313-316. doi:10.1016/0014-2999(80)90515-4

37. Dziennis S, Van Etten RA, Pahl HL, Morris DL, Rothstein TL, Blosch CM, Perlmutter RM, Tenen DG (1995) The CD11b promoter directs high-level expression of reporter genes in macrophages in transgenic mice. Blood 85 (2):319-329

38. Hu LY, Zhou Y, Cui WQ, Hu XM, Du LX, Mi WL, Chu YX, Wu GC, Wang YQ, Mao-Ying QL (2018) Triggering receptor expressed on myeloid cells 2 (TREM2) dependent microglial activation promotes cisplatin-induced peripheral neuropathy in mice. Brain Behav Immun 68:132-145. doi:10.1016/j.bbi.2017.10.011

39. Huo J, Ma R, Chai X, Liang HJ, Jiang P, Zhu XL, Chen X, Su BX (2019) Inhibiting a spinal cord signaling pathway protects against ischemia injury in rats. J Thorac Cardiovasc Surg 157 (2):494503.e491. doi:10.1016/j.jtcvs.2018.07.045

40. Devanney NA, Stewart AN, Gensel JC (2020) Microglia and macrophage metabolism in CNS injury and disease: The role of immunometabolism in neurodegeneration and neurotrauma. Exp Neurol 329:113310. doi:10.1016/j.expneurol.2020.113310

41. Krasemann S, Madore C, Cialic R, Baufeld C, Calcagno N, El Fatimy R, Beckers L, O'Loughlin E, Xu Y, Fanek Z, Greco DJ, Smith ST, Tweet G, Humulock Z, Zrzavy T, Conde-Sanroman P, Gacias M, Weng Z, Chen H, Tjon E, Mazaheri F, Hartmann K, Madi A, Ulrich JD, Glatzel M, Worthmann A, Heeren J, Budnik B, Lemere C, Ikezu T, Heppner FL, Litvak V, Holtzman DM, Lassmann H, Weiner HL, Ochando J, Haass C, Butovsky O (2017) The TREM2-APOE Pathway Drives the Transcriptional Phenotype of Dysfunctional Microglia in Neurodegenerative Diseases. Immunity 47 (3):566-581 e569. doi:10.1016/j.immuni.2017.08.008

42. Chen S, Peng J, Sherchan P, Ma Y, Xiang S, Yan F, Zhao H, Jiang Y, Wang N, Zhang JH, Zhang H (2020) TREM2 activation attenuates neuroinflammation and neuronal apoptosis via PI3K/Akt pathway after intracerebral hemorrhage in mice. J Neuroinflammation 17 (1):168. doi:10.1186/s12974-020-01853-x

43. Boza-Serrano A, Ruiz R, Sanchez-Varo R, Garcia-Revilla J, Yang Y, Jimenez-Ferrer I, Paulus A, Wennstrom M, Vilalta A, Allendorf D, Davila JC, Stegmayr J, Jimenez S, Roca-Ceballos MA, Navarro- 
Garrido V, Swanberg M, Hsieh CL, Real LM, Englund E, Linse S, Leffler H, Nilsson UJ, Brown GC, Gutierrez A, Vitorica J, Venero JL, Deierborg T (2019) Galectin-3, a novel endogenous TREM2 ligand, detrimentally regulates inflammatory response in Alzheimer's disease. Acta Neuropathol 138 (2):251273. doi:10.1007/s00401-019-02013-z

44. DiSabato DJ, Quan N, Godbout JP (2016) Neuroinflammation: the devil is in the details. J Neurochem 139 Suppl 2:136-153. doi:10.1111/jnc.13607

45. Becher B, Spath S, Goverman J (2017) Cytokine networks in neuroinflammation. Nat Rev Immunol 17 (1):49-59. doi:10.1038/nri.2016.123

46. Sandireddy R, Yerra VG, Areti A, Komirishetty P, Kumar A (2014) Neuroinflammation and oxidative stress in diabetic neuropathy: futuristic strategies based on these targets. Int $\mathrm{J}$ Endocrinol 2014:674987. doi:10.1155/2014/674987

47. Ji RR, Xu ZZ, Gao YJ (2014) Emerging targets in neuroinflammation-driven chronic pain. Nat Rev Drug Discov 13 (7):533-548. doi:10.1038/nrd4334

48. Srodulski S, Sharma S, Bachstetter AB, Brelsfoard JM, Pascual C, Xie XS, Saatman KE, Van Eldik LJ, Despa $F$ (2014) Neuroinflammation and neurologic deficits in diabetes linked to brain accumulation of amylin. Mol Neurodegener 9:30. doi:10.1186/1750-1326-9-30

49. Ellis A, Bennett DL (2013) Neuroinflammation and the generation of neuropathic pain. Br J Anaesth 111 (1):26-37. doi:10.1093/bja/aet128

50. Wu M, Liao M, Huang R, Chen C, Tian T, Wang H, Li J, Li J, Sun Y, Wu C, Li Q, Xiao X (2020)

Hippocampal overexpression of TREM2 ameliorates high fat diet induced cognitive impairment and modulates phenotypic polarization of the microglia. Genes \& Diseases.

doi:https://doi.org/10.1016/j.gendis.2020.05.005

51. Guan Z, Kuhn JA, Wang X, Colquitt B, Solorzano C, Vaman S, Guan AK, Evans-Reinsch Z, Braz J, Devor M, Abboud-Werner SL, Lanier LL, Lomvardas S, Basbaum Al (2016) Injured sensory neuronderived CSF1 induces microglial proliferation and DAP12-dependent pain. Nat Neurosci 19 (1):94101. doi:10.1038/nn.4189

\section{Figures}


A

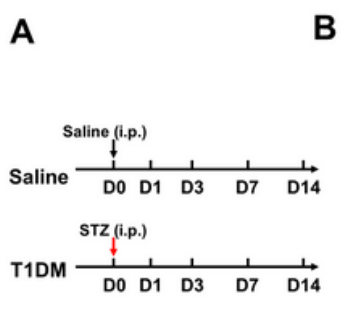

E
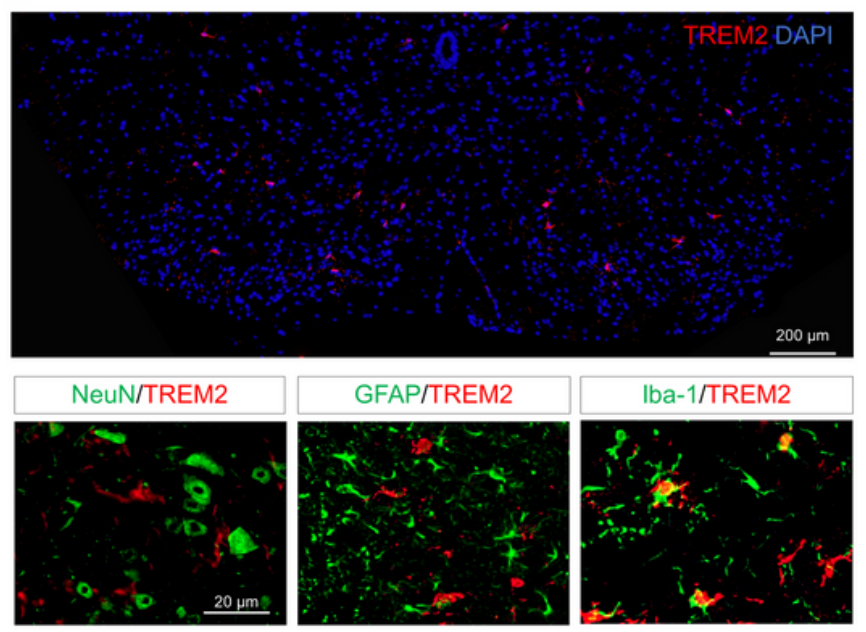

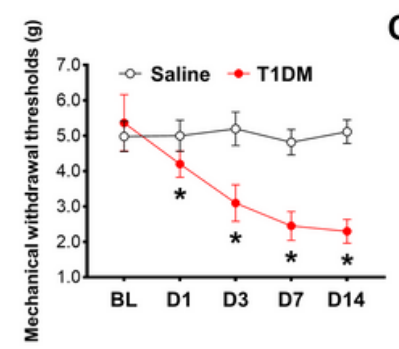

GFAP/TREM2

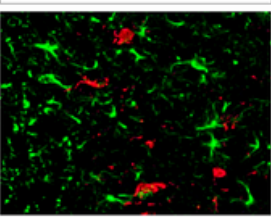

Iba-1/TREM2

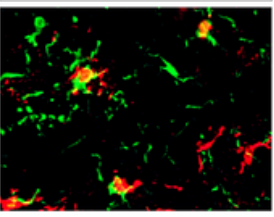

C

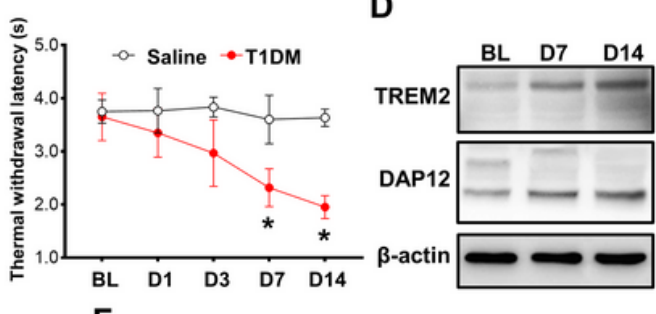

$\mathbf{F}$
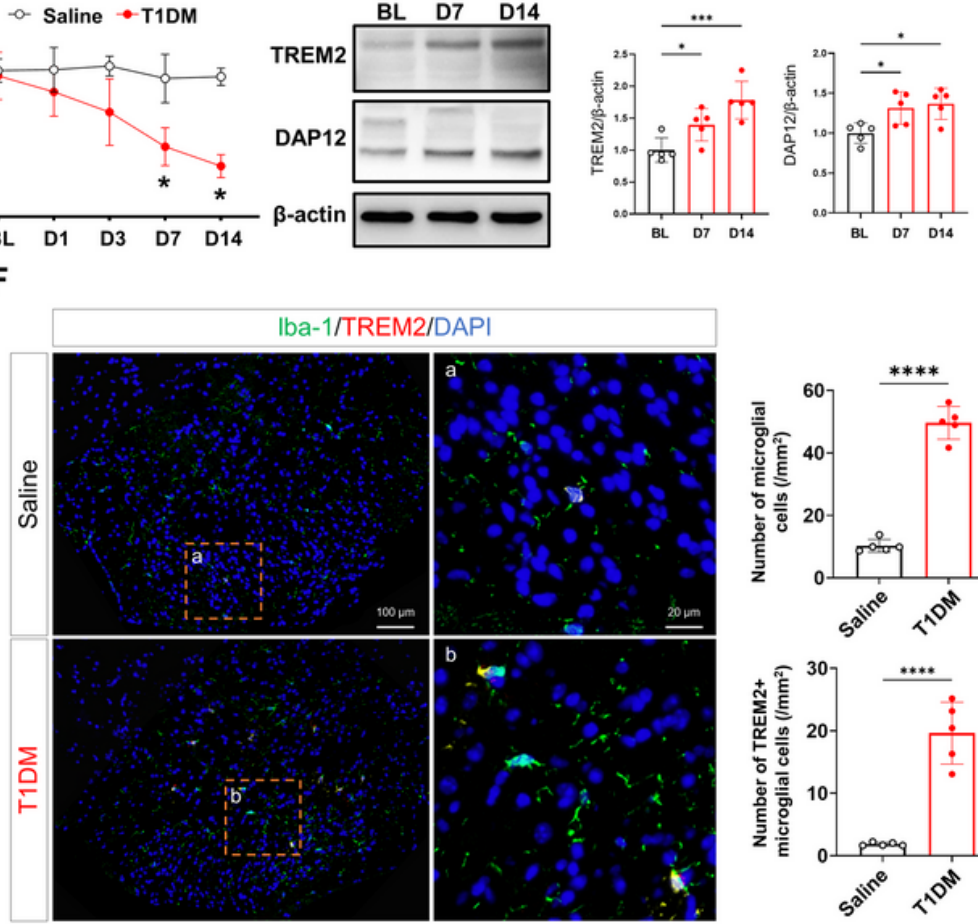

.

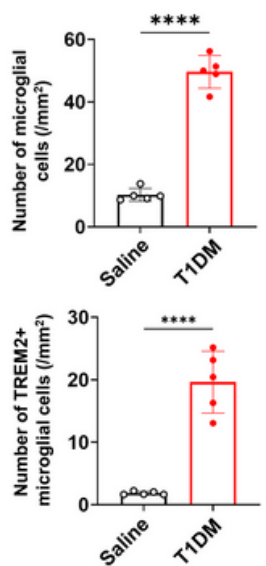

\section{Figure 1}

The spinal TREM2 increased along with the development of PDN. (A) schematic illustration of the group assignment. (B) Streptozotocin (STZ) injection-induced diabetic mice (T1DM) displayed mechanical allodynia (reduction in PMWTs). The PMWTs were evaluated by electronic von Frey test before and after STZ injection in Control or T1DM mice. Two-way ANOVA showed a significant difference between Saline and T1DM groups, F1, $10=144.4, \mathrm{P}<0.0001, \mathrm{n}=6$; Bonferroni post hoc analysis revealed a significant difference between groups on D1, D3, D7, and D14 ( $\left.{ }^{*} P<0.05\right)$. mean \pm SD. (C) The STZ injection-induced diabetic mice developed thermal hypersensitivity (decrease in PTWL). The PTWL was assessed by a thermal paw stimulation system before and after STZ injection in Saline or T1DM mice. Two-way ANOVA showed a significant difference between Saline and T1DM groups, F1, $10=43.93, P<0.0001, n=6$; Bonferroni post hoc analysis revealed a significant difference between groups on D7 and D14 $(* P<0.05)$. mean \pm SD. (C) Western blot showing the spinal TREM2 and DAP12 protein expression on baseline (BL), D7, and D14 after STZ injection. * $\mathrm{P}<0.05$ and $* \star \star ~ P<0.001$ vs. the BL. $n=5$. mean \pm SD. (D) Representative immunofluorescence images showing the cellular expression of TREM2 on D14 after STZ injection. The above panel indicates the distribution of TREM2+ (red) cells in the dorsal horn of T1DM mice (Scale bar=100 $\mu \mathrm{m}$ ). The bottom panels show the double-staining of TREM2 (red) with NeuN (the neuron marker, green), GFAP (the astrocyte marker, green), and Iba-1 (the microglia marker, green), respectively (Scale bar=20 $\mu \mathrm{m}$ ). The sections were counterstained with DAPI (blue). (E) Fluorescent results showing Iba-1+ (green) and TREM2+ (red) cells in the dorsal horn of the Control and T1DM mice on D14 after STZ injection. The scale bar of the left panels is $100 \mu \mathrm{m}$. The yellow dotted boxes (a and b) are zoomed-in in

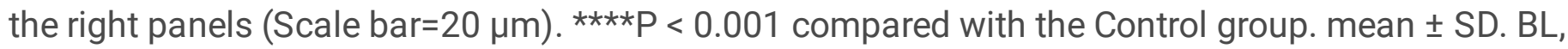
baseline; DAPI, 4',6-diamidino-2-phenylindole; GFAP, glial fibrillary acidic protein; Iba-1, ionized calcium- 
binding adaptor protein-1; PDN, painful diabetic neuropathy; PMWT, paw mechanical withdrawal threshold; PTWL, paw thermal withdrawal latency; STZ, streptozotocin; T1DM, type 1 diabetes mellitus; TREM2, triggering receptor expressed on myeloid cells 2.

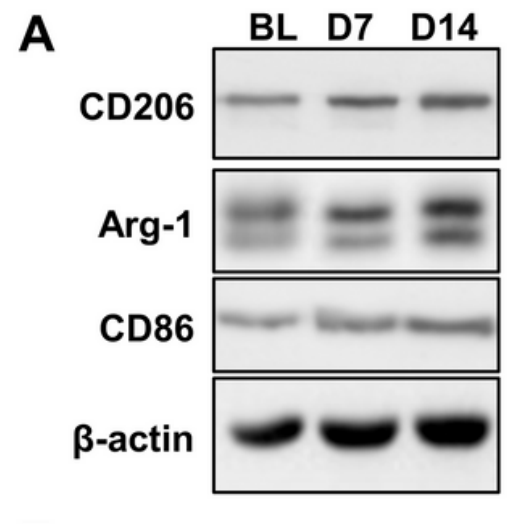

E

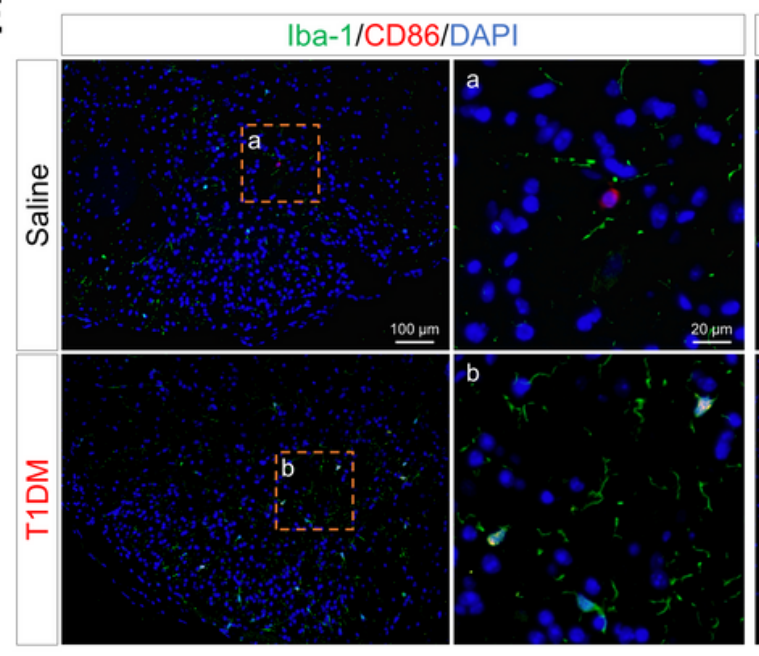

H

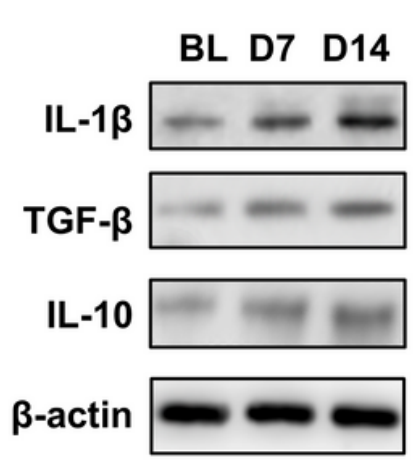

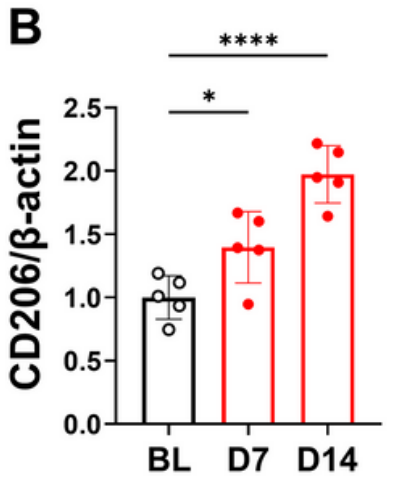

BL D7 D14

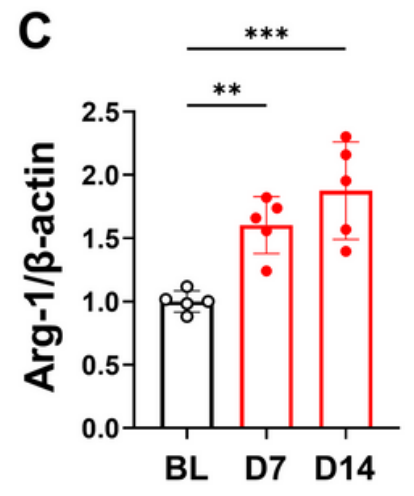

|ba-1/CD206/DAP|
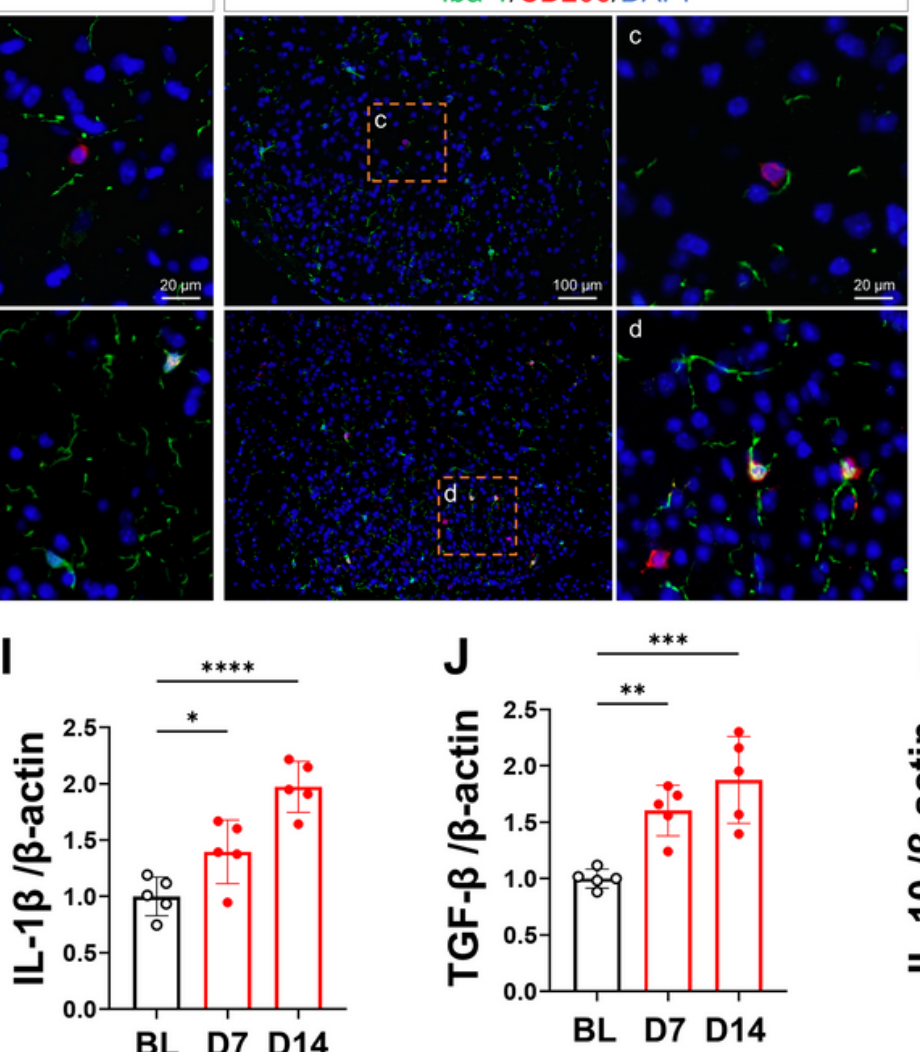

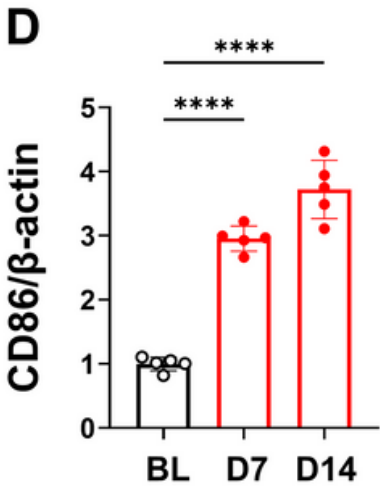

$\mathbf{F}$
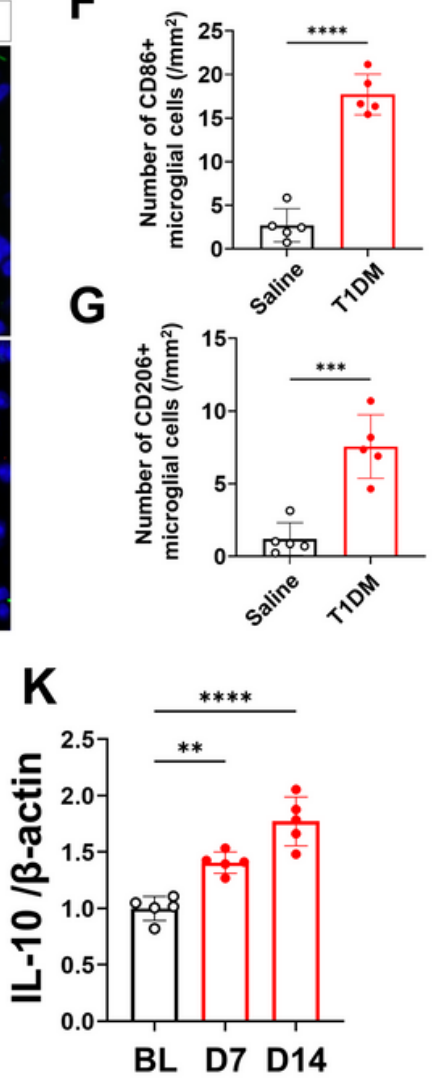

Figure 2

M1 and M2 microglia both increased in parallel to the development of PDN. (A) Western blot indicating the increased protein expression of CD206, Arg-1, and CD86 in the spinal cord of the T1DM mice on BL, D7, and D14 after STZ injection. (B-D) The semi-quantity analysis of the spinal protein expression of CD206, Arg-1, and CD86. $n=5$. ${ }^{*} P<0.05, * \star P<0.01, * \star * P<0.001$, or $* \star \star * P<0.0001$ compared with the baseline. (E) Fluorescent images showing lba-1+ (green)/CD86+ (red) (M1 microglia) and Iba-1 (green)/CD206 (red) (M2 microglia) double-staining in the dorsal horn of the Saline and T1DM mice. The nuclei were counterstained with DAPI (blue). The scale bar of the left panels is $100 \mu \mathrm{m}$. The yellow dotted 
boxes (a-d) are zoomed-in in the right panels (Scale bar $=50 \mu \mathrm{m}) .(F, G)$ The statistical analysis of the CD86+lba-1+ and CD206+lba-1+ cells in the dorsal horn of the Control and T1DM mice. $n=5 . * \star \star \star P<$ 0.001 compared with the Control group. $(H)$ Western blot indicating the protein expression of IL-1 $\beta$, TGF- $\beta$, and IL-10 in the spinal cord of the Control and T1DM mice. (I-K) The analysis of the spinal protein expression of IL- $1 \beta$, TGF- $\beta$, and IL-10. $n=5$. ${ }^{*} P<0.05$, ${ }^{*} P<0.01, * \star * P<0.001$ vs. the baseline. All the data were expressed as mean $\pm S D$. Arg-1, arginase-1; BL, baseline; DAPI, 4',6-diamidino-2-phenylindole; lba-1, ionized calcium-binding adaptor protein-1; IL-1 $\beta$, interleukin 1 $\beta$; IL-10, interleukin 10; STZ, Streptozotocin; T1DM, type 1 diabetes mellitus; PDN, painful diabetic neuropathy; TGF- $\beta$, transforming growth factor $\beta$; TREM2, triggering receptor expressed on myeloid cells 2 .

A

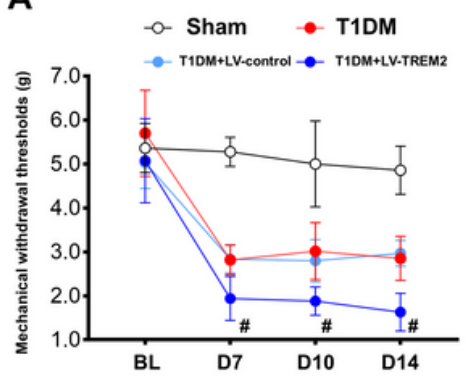

E

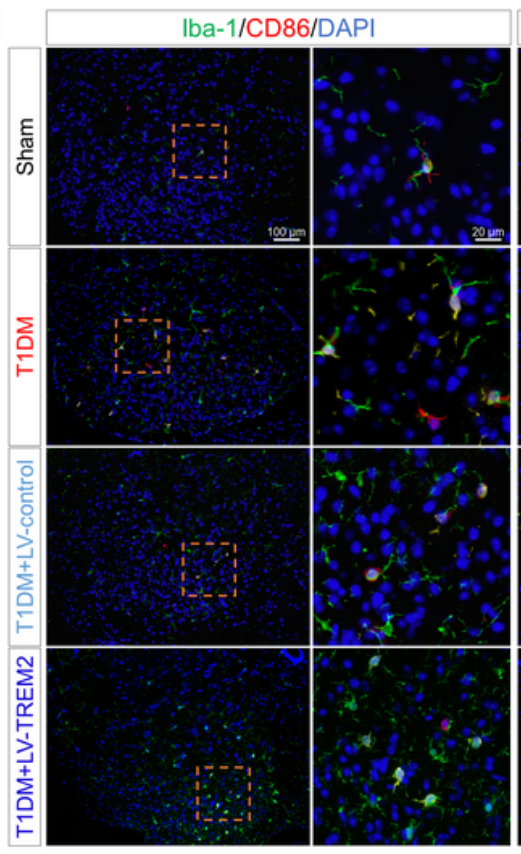

B
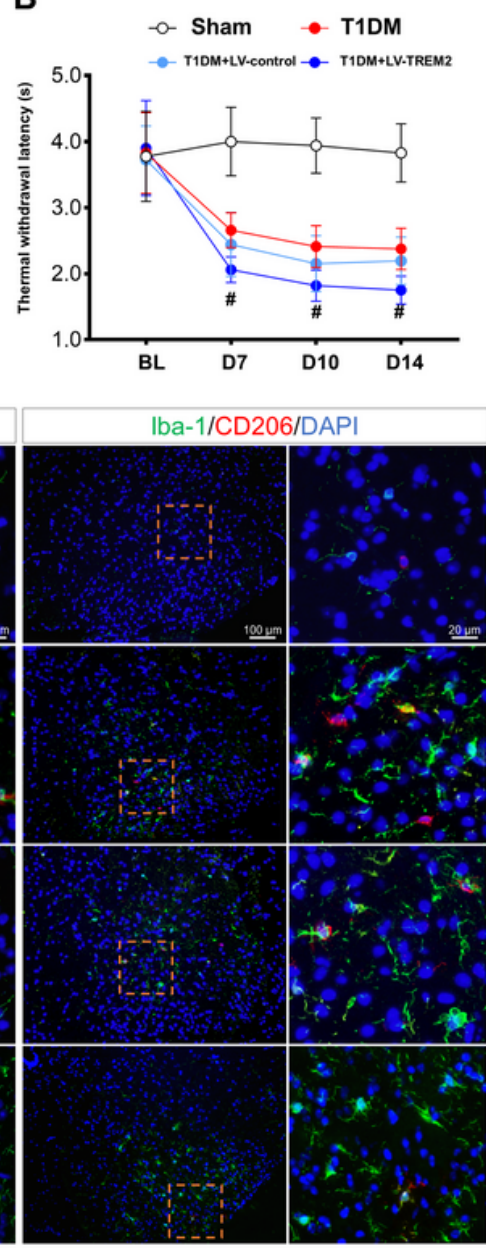

C

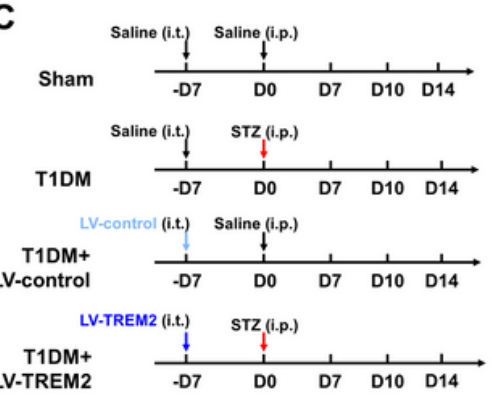

$\mathbf{F}$
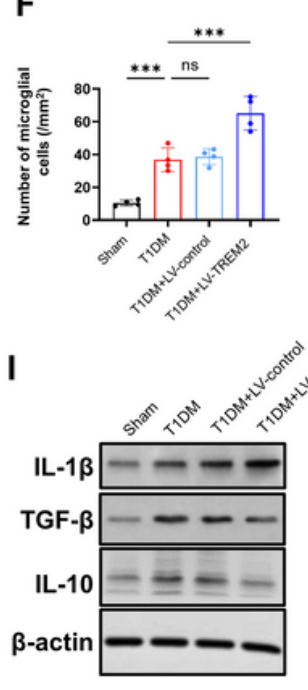

G

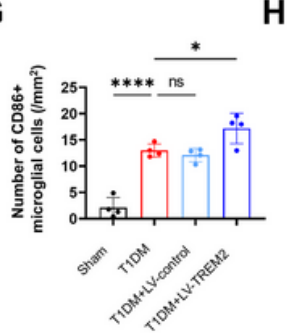

D

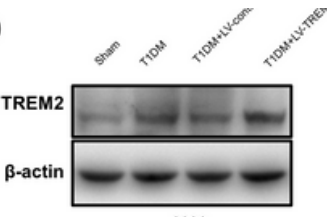

...**

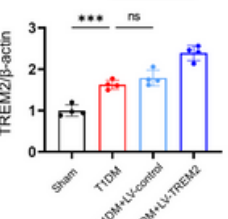

H
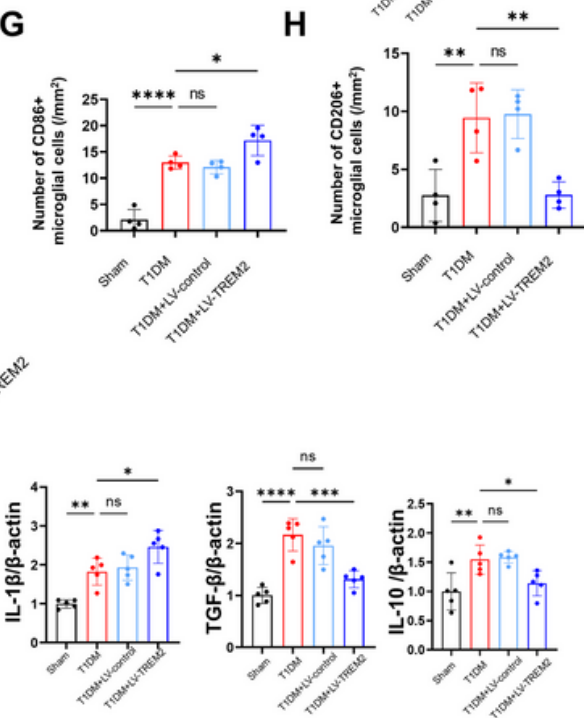

Figure 3

Overexpression of TREM2 in microglia aggravated PDN, induced microglial M1 polarization, and reduced $M 2$ response in mice. (A) Intrathecal administration of microglia-specific TREM2 overexpression lentiviral vectors (LV-TREM2, 5×107 TU, 7 days before STZ injection) exacerbated mechanical allodynia (reduction in PMWTs) in the diabetic mice. mean \pm SD. T1DM vs. T1DM+LV-TREM2: $F 1,14=35.21, P<0.0001, n=8$, two-way ANOVA; Bonferroni post hoc analysis revealed a significant difference between T1DM and T1DM+LV-TREM2 on D7, D10, and D14 (\#P<0.05). (B) Intrathecal administration of microglia-specific TREM2 overexpression lentiviral vectors (LV-TREM2, 5×107 TU, 7 days before STZ injection) aggravated 
thermal hypersensitivity (decrease in PMWTs) in the diabetic mice. mean \pm SD. T1DM vs. T1DM+LVTREM2: $F 1,14=14.27, P=0.0020, n=8$, two-way ANOVA; Bonferroni post hoc analysis revealed a significant difference between T1DM and T1DM+LV-TREM2 on D7, D10, and D14 (\#P<0.05) (C) Schematic illustration of the assignment and treatment in Sham, T1DM, T1DM+LV-control, and T1DM+LV-TREM2 groups. (D) Western blot showing the effect of LV-control or LV-TREM2 intrathecal administration on TREM2 protein expression in the lumbar spinal cord on D14 after STZ injection.

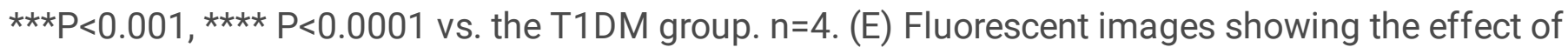
microglial TREM2 overexpression on the number of Iba-1+ (green)/CD86+ (red) and Iba-1+ (green)/CD206+ (red) cells in the dorsal horn on D14 after STZ injection. The nuclei were counterstained with DAPI (blue). The scale bar of the left panels is $100 \mu \mathrm{m}$. The yellow dotted boxes (a-d) are zoomed-in in the right panels (Scale bar $=20 \mu \mathrm{m}) .(\mathrm{F}-\mathrm{H})$ The analysis of the CD86+lba- $1+$ and CD206+lba- $1+$ cells in the dorsal horn on D14 after STZ injection. mean \pm SD. $n=5$. ${ }^{* *} P<0.01,{ }^{* \star *} P<0.001$ vs. the T1DM group. (I) Representative graphs of western blot indicating the protein expression of IL-1 $\beta$, TGF- $\beta$, and IL-10 in the spinal cord on D14 after STZ injection. $n=5 .{ }^{*} P<0.05,{ }^{*} P<0.01$, $* \star * ~ P<0.001$ vs. the T1DM group. Arg- 1 , arginase-1; BL, baseline; DAPI, 4',6-diamidino-2-phenylindole; Iba-1, ionized calcium-binding adaptor protein-1; LV-control, control lentiviral vectors; LV-TREM2, microglia specific TREM2 overexpression lentiviral vectors; ns, not significant; STZ, Streptozotocin; T1DM, type 1 diabetes mellitus; TREM2, triggering receptor expressed on myeloid cells 2 . 


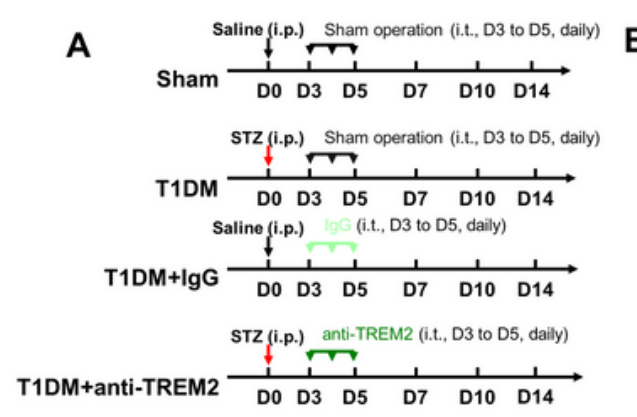

D

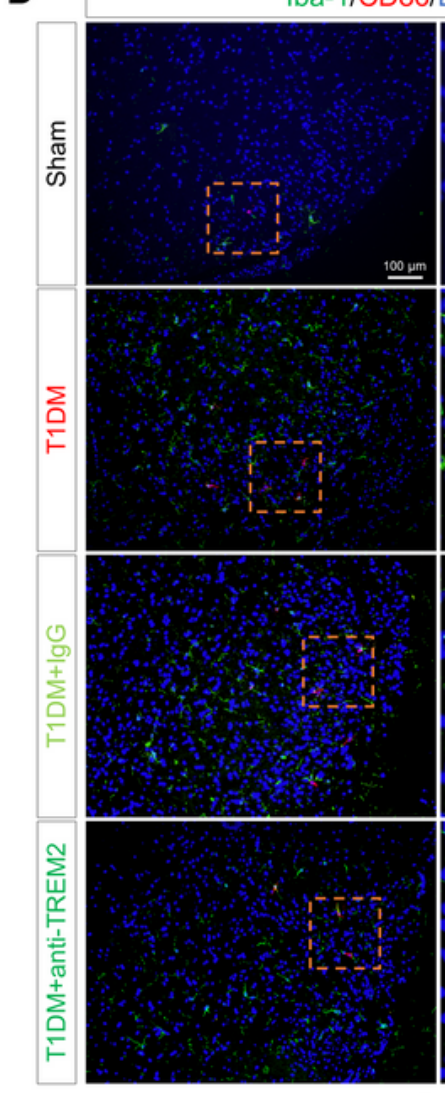

H

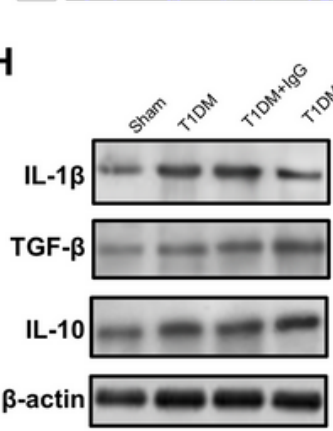

\begin{abstract}
年
\end{abstract}
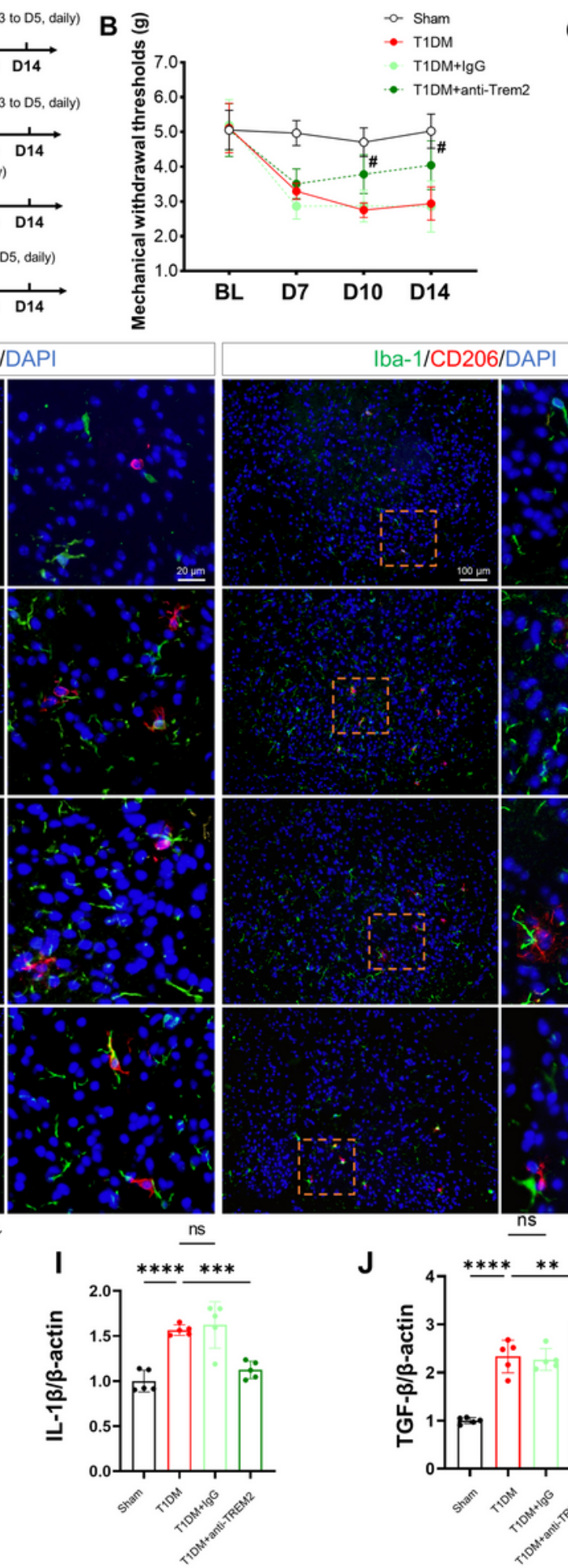

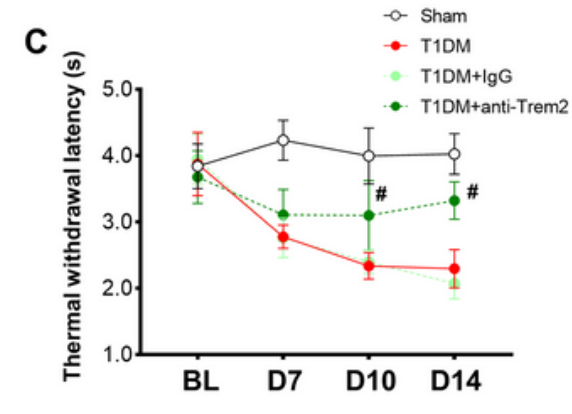

E
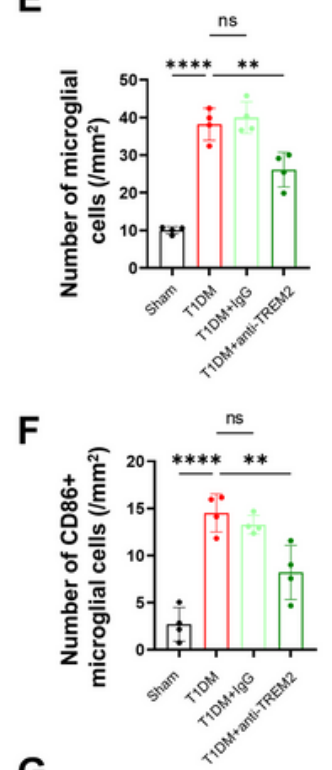

G
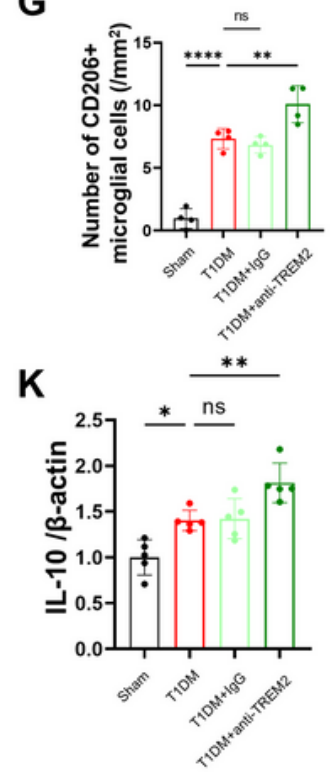

\section{Figure 4}

Intrathecal treatment with anti-TREM2 neutralizing antibody ameliorated PDN, suppressed microglial M1 polarization, and facilitated M2 polarization in mice. (A) Schematic illustration of the assignment and treatment in Sham, T1DM, T1DM+lgG, and T1DM+anti-TREM2 groups. (B) Intrathecal treatment with antiTREM2 neutralizing antibody ( $1 \mu \mathrm{g} / \mathrm{d}$, from D3 to D5 after STZ injection) improved mechanical allodynia (increase in PMWTs) in the diabetic mice. mean \pm SD. T1DM vs. T1DM+anti-TREM2: F1, $16=23.17$, 
$\mathrm{P}=0.0002, \mathrm{n}=9$, two-way ANOVA. Bonferroni post hoc analysis revealed a significant difference between T1DM and T1DM+anti-TREM2 on D10 and D14 (\#P<0.05). (C) Intrathecal treatment with anti-TREM2 neutralizing antibody ( $1 \mu \mathrm{g} / \mathrm{d}$, from D3 to D5 after STZ injection) attenuated thermal hypersensitivity (elevation in PMWTs) in diabetic mice. mean \pm SD. T1DM vs. T1DM+anti-TREM2: PTWL, F1, $16=19.65$, $\mathrm{P}=0.0004, \mathrm{n}=9$, two-way ANOVA. Bonferroni post hoc analysis revealed a significant difference between T1DM and T1DM+anti-TREM2 on D10 and D14 (\#P<0.05). (D) Fluorescent images showing the effect of intrathecal treatment with anti-TREM2 neutralizing antibody on the number of Iba- $1+$ (green)/CD $86+$ (red) and Iba-1+ (green)/CD206+ (red) cells in the dorsal horn on D14 after STZ injection. The nuclei were counterstained with DAPI (blue). The scale bar of the left panels is $100 \mu \mathrm{m}$. The yellow dotted boxes (a-d) are zoomed-in in the right panels (Scale bar $=20 \mu \mathrm{m})$. (E-G) The analysis of the CD86+lba-1+ and CD206+lba-1+ cells in the dorsal horn on D14 after STZ injection. mean \pm SD. $n=5 .{ }^{* * P<0.01, ~}{ }^{\star \star \star} P<0.001$ vs. the T1DM group. $(H)$ Representative graphs of western blot indicating the protein expression of IL-1 $\beta$, TGF- $\beta$, and IL-10 in the spinal cord on D14 after STZ injection. (I-K) The semi-quantity analysis of the spinal protein expression of IL-1 $\beta$, TGF- $\beta$, and IL-10. $n=5$. ${ }^{*} P<0.05$, ${ }^{\star * P} P<0.01$, $* \star * ~ P<0.001$ vs. the T1DM group. anti-TREM2, anti-TREM2 antibody; Arg-1, arginase-1; BL, baseline; DAPI, 4',6-diamidino-2phenylindole; Iba-1, ionized calcium-binding adaptor protein-1; IL-1 $\beta$, interleukin 1 $\beta$; IL-10, interleukin 10; TGF- $\beta$, transforming growth factor $\beta$; IgG, control rabbit isotype IgG; STZ, streptozotocin; T1DM, type 1 diabetes mellitus; PDN, painful diabetic neuropathy; PMWT, paw mechanical withdrawal threshold; PTWL, paw thermal withdrawal latency; Sham: mice receiving only intrathecal puncture; TREM2, triggering receptor expressed on myeloid cells 2 . 
A

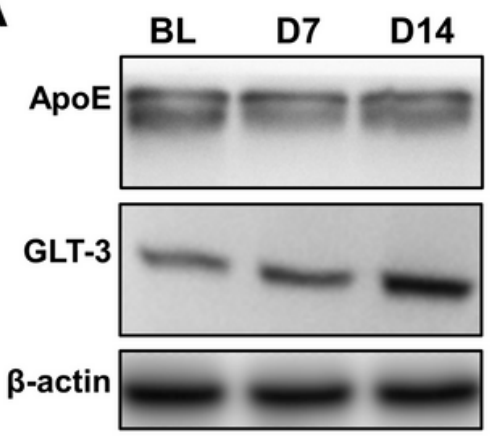

E

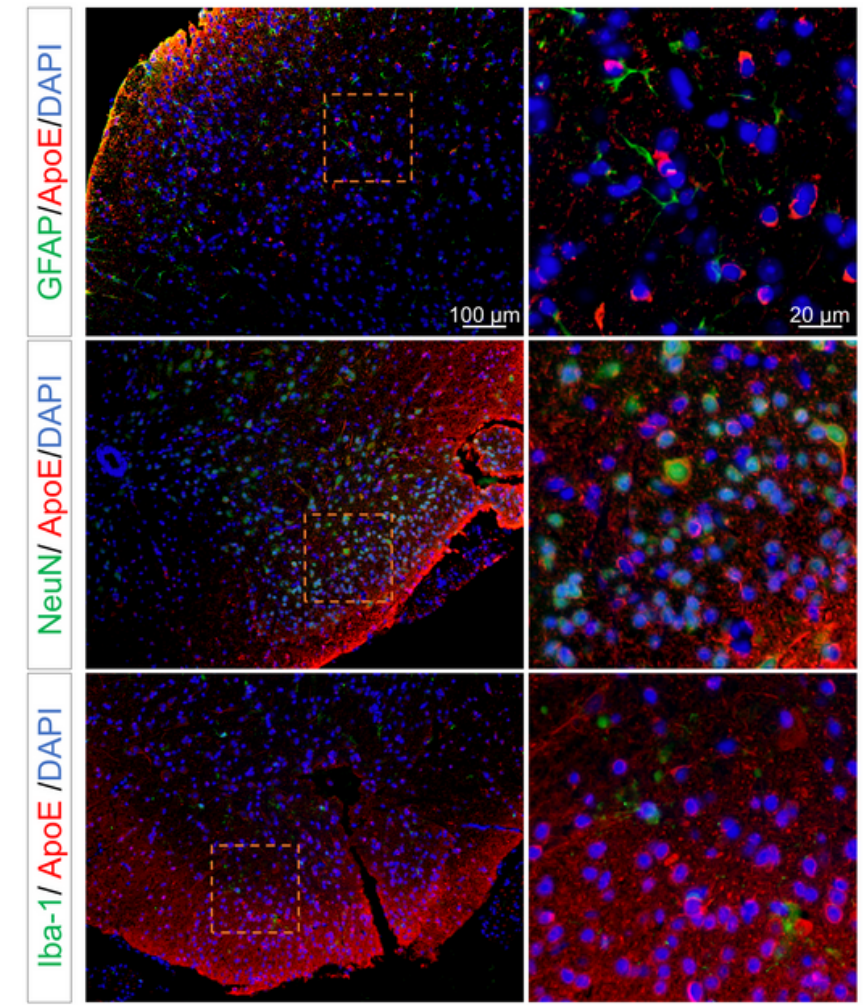

B

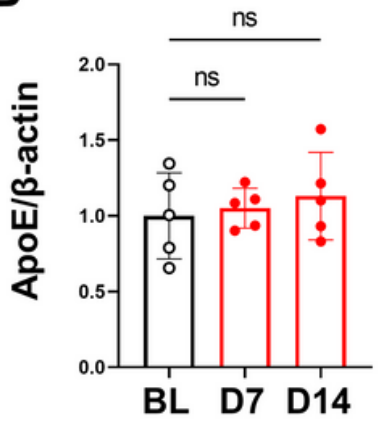

C

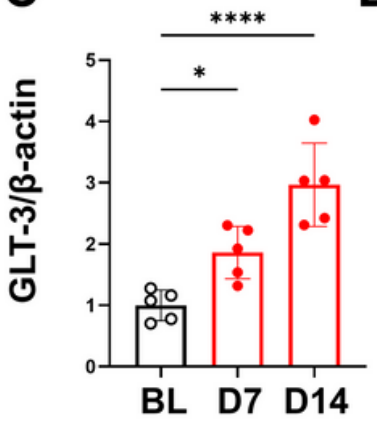

D IP: TREM2 IgG Saline T1DM

IB: GLT-3

IB: ApoE

IB: TREM2

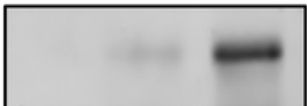

$\mathbf{F}$

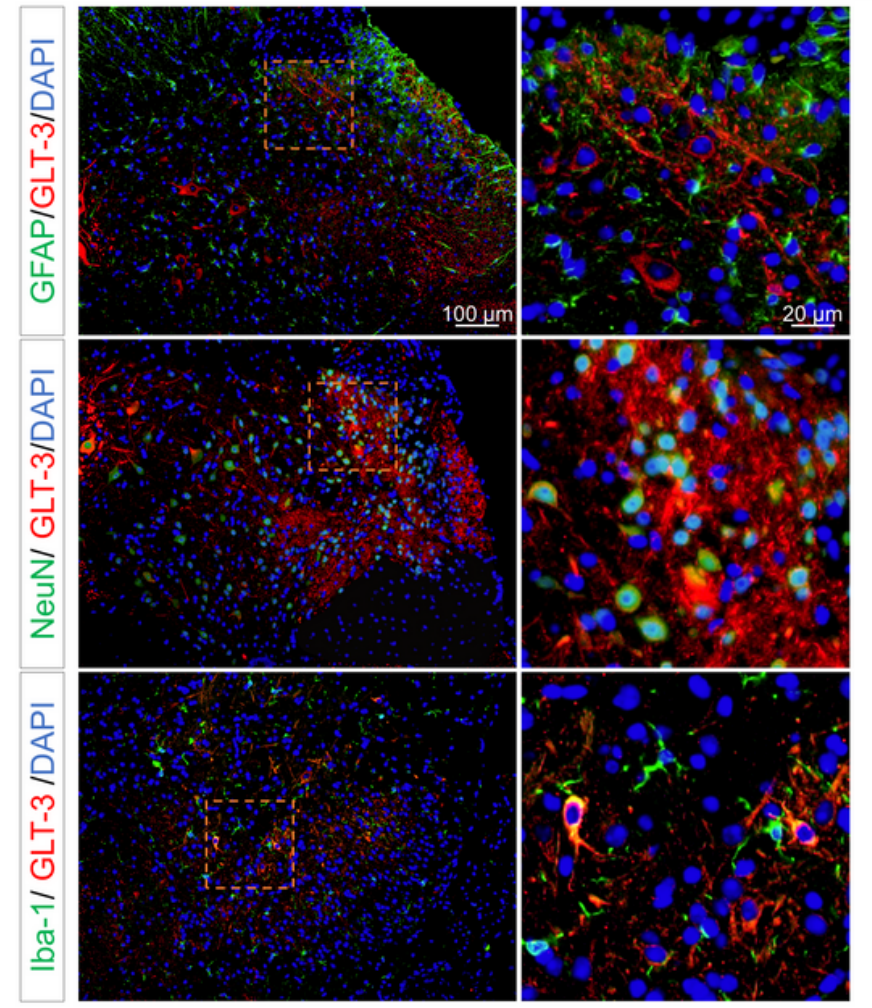

\section{Figure 5}

GLT-3 was the potential ligand of microglial TREM2 receptor in facilitating the progression of PDN. (A) Western blot showing the spinal ApoE and GLT-3 protein expression on the baseline, D7, and D14 after STZ injection. $(B, C)$ The analysis of the protein expression of ApoE and GLT-3 in the spinal cord. ${ }^{*} P<0.05$, $\star \star \star P<0.001$ vs. the baseline. $n=5$. mean \pm SD. (D) Immunoprecipitation of ApoE or GLT-3 with TREM2. The levels of immunoprecipitated ApoE or GLT-3 by anti-TREM2 antibodies were normalized to the corresponding input. (E) Representative immunofluorescence images showing the double-staining of ApoE (red) with NeuN (green), GFAP (green), and lba-1 (green). The scale bar of the left panels is $100 \mu \mathrm{m}$. The yellow dotted boxes are zoomed-in in the right panels (Scale bar $=20 \mu \mathrm{m}$ ). The sections were counterstained with DAPI (blue). (F) Fluorescent images showing the double-staining of GLT-3 (red) with NeuN (green), GFAP (green), and lba-1 (green). The scale bar of the left panels is $100 \mu \mathrm{m}$. The right panels are the corresponding magnified images indicated by the yellow dotted boxes in the left panels. The sections were counterstained with DAPI (blue). APOE, apolipoprotein E; BL, baseline; DAPI, 4',6-diamidino- 
2-phenylindole; GFAP, glial fibrillary acidic protein; GLT-3, galectin-3; Iba-1, ionized calcium-binding adaptor protein-1; T1DM, type 1 diabetes mellitus; TREM2, triggering receptor expressed on myeloid cells 2.

A

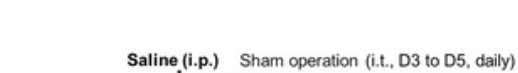

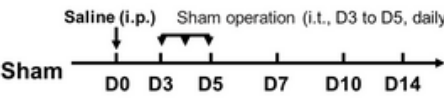

STZ (i.p.) Sham operation (i.t., D3 to D5, daily)

T1DM

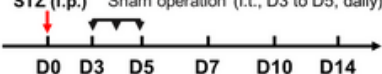

Saline (i.p.) $\quad$ (i.t., D3 to D5, daily)
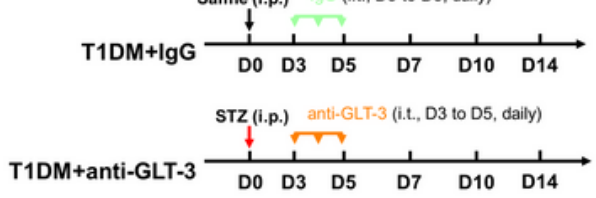

B

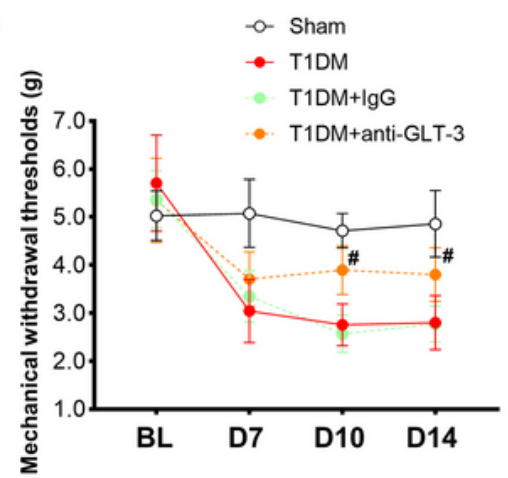

D

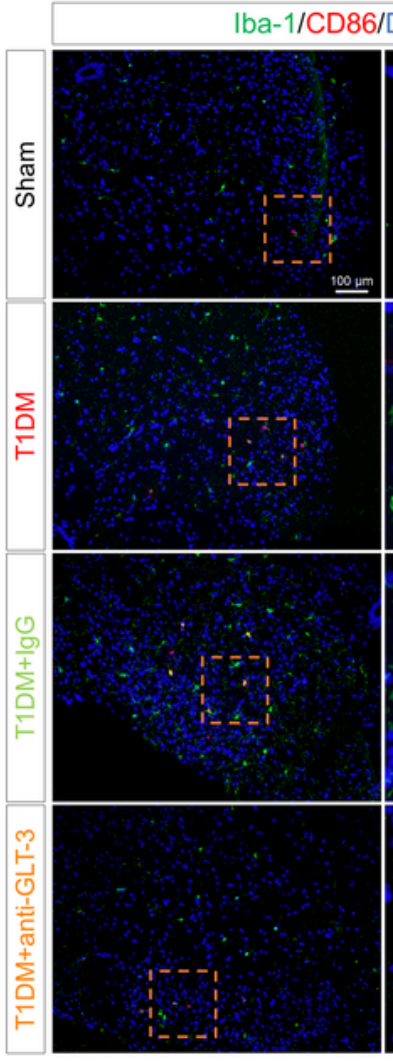

H

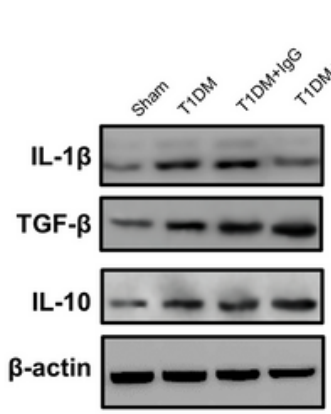

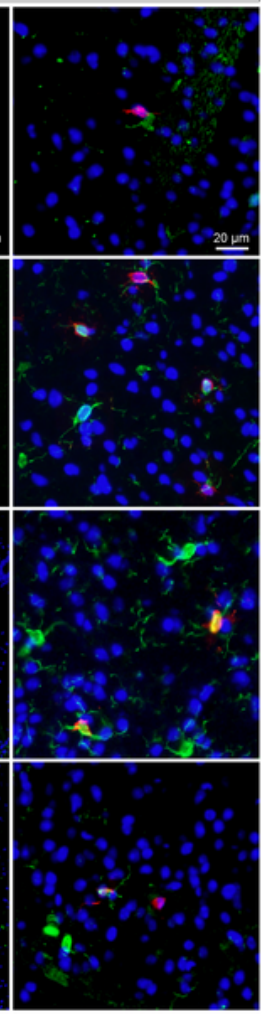

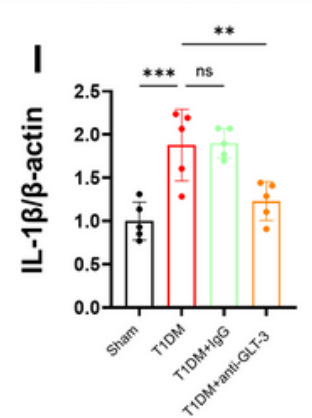

C

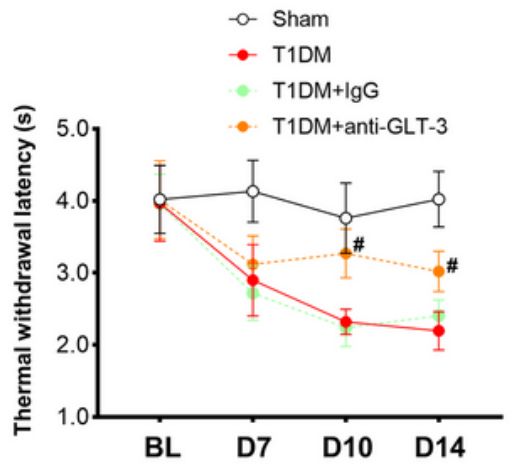

E

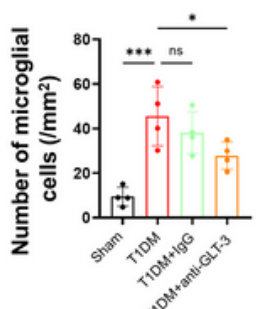

$\mathbf{F}$
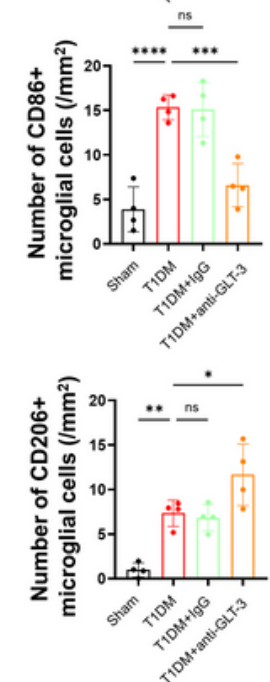

K

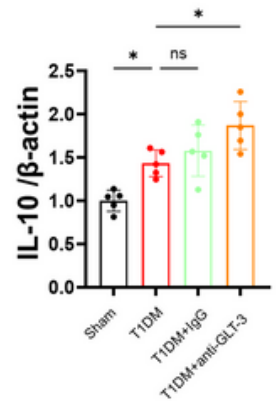

\section{Figure 6}

Intrathecal treatment with anti-GLT-3 neutralizing antibody attenuated PDN, inhibited microglial M1 polarization, and augmented M2 polarization in mice. (A) Schematic illustration of the assignment and treatment in Sham, T1DM, T1DM+IgG, and T1DM+anti-GLT-3 groups. (B) Intrathecal treatment with anti- 
GLT-3 neutralizing antibody ( $1 \mu \mathrm{g} / \mathrm{d}$, from D3 to D5 after STZ injection) improved mechanical allodynia (increase in PMWTs) in the T1DM+anti-GLT-3 mice. mean \pm SD. T1DM vs. T1DM+anti-GLT-3: F1, 16 $=16.09, P=0.001, n=9$, two-way ANOVA. Bonferroni post hoc analysis revealed a significant difference between T1DM and T1DM+anti-GLT-3 on D10 and D14 (\#P<0.05). (C) Intrathecal treatment with anti-GLT3 neutralizing antibody ( $1 \mu \mathrm{g} / \mathrm{d}$, from D3 to D5 after STZ injection) attenuated thermal hypersensitivity (elevation in PMWTs) in T1DM+anti-GLT-3 mice. mean \pm SD. T1DM vs. T1DM+anti-GLT-3: F1, $16=40.63$, $\mathrm{P}<0.0001, \mathrm{n}=9$, two-way ANOVA. Bonferroni post hoc analysis revealed a significant difference between T1DM and T1DM+anti-GLT-3 on D10 and D14 (\#P<0.05). (D) Fluorescent images showing the effect of intrathecal treatment with anti-GLT-3 neutralizing antibody on the number of Iba-1+ (green)/CD86+ (red) and Iba-1+ (green)/CD206+ (red) cells in the dorsal horn on D14 after STZ injection. The nuclei were counterstained with DAPI (blue). The scale bar of the left panels is $100 \mu \mathrm{m}$. The yellow dotted boxes (a-d) are zoomed-in in the right panels (Scale bar $=20 \mu \mathrm{m}$ ). (E-G) The analysis of the CD $86+\mid \mathrm{lba}-1+$ and CD206+lba- $1+$ cells in the dorsal horn on D14 after STZ injection. mean $\pm S D . n=5 .{ }^{* \star P} P<0.01,{ }^{* \star *} P<0.001$ vs. the T1DM group. $(H)$ Representative graphs of western blot indicating the protein expression of IL-1 $\beta$, TGF- $\beta$, and IL-10 in the spinal cord on D14 after STZ injection. (I-K) The semi-quantity analysis of the spinal protein expression of IL-1 $\beta$, TGF- $\beta$, and IL-10. $n=5$. ${ }^{*} P<0.05$, ${ }^{\star *} P<0.01$, ${ }^{\star \star *} P<0.001$ vs. the T1DM group. Arg-1, arginase-1; BL, baseline; DAPI, 4',6-diamidino-2-phenylindole; GLT-3, galectin-3; Iba-1, ionized calcium-binding adaptor protein-1; IgG, control rabbit isotype IgG; IL-1 $\beta$, interleukin 1 $\beta$; IL-10, interleukin 10; STZ, Streptozotocin; T1DM, type 1 diabetes mellitus; PDN, painful diabetic neuropathy; PMWT, paw mechanical withdrawal threshold; PTWL, paw thermal withdrawal latency; TGF- $\beta$, transforming growth factor $\beta$; TREM2, triggering receptor expressed on myeloid cells 2 , anti-GLT-3, anti-GLT-3 antibody. 